\title{
COMPARING SIMULATIONS OF AGN FEEDBACK
}

\author{
Mark L. A. Richardson ${ }^{1,2}$, Evan Scannapieco ${ }^{2}$, Julien Devriendt ${ }^{1,3}$, Adrianne Slyz $^{1}$, Robert J. Thacker ${ }^{4}$, \\ YOHAN Dubois ${ }^{5,6}$, JAMES WURSTER ${ }^{4,7,8}$, AND JOSEPH SILK S $^{1,5,6,9}$ \\ ${ }^{1}$ Sub-department of Astrophysics, University of Oxford, Keble Road, Oxford, OX1 3RH, UK \\ ${ }^{2}$ School of Earth and Space Exploration, Arizona State University, Tempe, AZ 85287, USA \\ ${ }^{3}$ Observatoire de Lyon, UMR 5574, 9 avenue Charles André, F-69561 Saint Genis Laval, France \\ ${ }^{4}$ Department of Astronomy \& Physics, Saint Mary's University, Halifax, NS, B3L 3C3, Canada \\ ${ }^{5}$ Sorbonne Universités, UPMC Univ Paris 06, UMR 7095, Institut d'Astrophysique de Paris, F-75014, Paris, France \\ ${ }^{6}$ CNRS, UMR 7095, Institut d'Astrophysique de Paris, F-75014, Paris, France \\ ${ }^{7}$ School of Physics and Astronomy, University of Exeter, Stocker Road, Exeter EX4 4QL, UK \\ ${ }^{8}$ Monash Centre for Astrophysics and School of Physics and Astronomy, Monash University, Victoria, 3800, Australia \\ ${ }^{9}$ Department of Physics and Astronomy, The Johns Hopkins University Homewood Campus, Baltimore, MD 21218, USA \\ Received 2016 February 5; revised 2016 April 26; accepted 2016 April 28; published 2016 July 5
}

\begin{abstract}
We perform adaptive mesh refinement (AMR) and smoothed particle hydrodynamics (SPH) cosmological zoom simulations of a region around a forming galaxy cluster, comparing the ability of the methods to handle successively more complex baryonic physics. In the simplest, non-radiative case, the two methods are in good agreement with each other, but the SPH simulations generate central cores with slightly lower entropies and virial shocks at slightly larger radii, consistent with what has been seen in previous studies. The inclusion of radiative cooling, star formation, and stellar feedback leads to much larger differences between the two methods. Most dramatically, at $z=5$, rapid cooling in the AMR case moves the accretion shock to well within the virial radius, while this shock remains near the virial radius in the SPH case, due to excess heating, coupled with poorer capturing of the shock width. On the other hand, the addition of feedback from active galactic nuclei (AGNs) to the simulations results in much better agreement between the methods. For our AGN model, both simulations display halo gas entropies of $100 \mathrm{keV} \mathrm{cm}{ }^{2}$, similar decrements in the star formation rate, and a drop in the halo baryon content of roughly $30 \%$. This is consistent with the AGN growth being self-regulated, regardless of the numerical method. However, the simulations with AGN feedback continue to differ in aspects that are not self-regulated, such that in SPH a larger volume of gas is impacted by feedback, and the cluster still has a lower entropy central core.
\end{abstract}

Key words: galaxies: active - galaxies: clusters: general - galaxies: evolution - galaxies: halos - methods: numerical

\section{INTRODUCTION}

In the hierarchical cold dark matter and dark energy $(\Lambda \mathrm{CDM})$ model for galaxy formation, matter condenses into small clumps that then merge to create increasingly massive objects over time. This model has provided several predictions that are in excellent agreement with observations (e.g., Spergel et al. 2007; Larson et al. 2011). For star formation to begin in $\Lambda \mathrm{CDM}$, the temperature of gas contained within condensed dark matter "halos" must cool sufficiently to allow the formation of galaxies. Because larger galaxies have more gravitational compression, and hence a higher temperature, they might be expected to take longer to cool and form stars, with the largest galaxies only now reaching significant star formation rates (SFRs).

Observational studies of SFRs, on the other hand, have found several surprising trends: the cosmic SFR density reaches a peak at $z \simeq 2,3$ to 5 billion years after the Big Bang and then decreases until the present (Hopkins \& Beacom 2006; Karim et al. 2011), SFRs peaked earlier in more massive galaxies and more recently in smaller galaxies (e.g., Guzman et al. 1997; Brinchmann \& Ellis 2000), and today SFRs are lower in more massive galaxies (Heavens et al. 2004; Panter et al. 2007). These trends, known collectively as "downsizing" (e.g., Cowie et al. 1996; Bauer et al. 2005; Panter et al. 2007; Karim et al. 2011), are clearly at odds with the naive predictions of the $\Lambda \mathrm{CDM}$ model.
A similar such discrepancy involves the properties of galaxy clusters, as constrained by high-resolution $\mathrm{X}$-ray and radio observations such as those from the Chandra X-ray Observatory and the Very Large Array. While many clusters appear to be quiescent, about a third show strong peaks in their central $\mathrm{X}$-ray surface brightness distributions, indicating that their gas is cooling rapidly (e.g., Fabian \& Nulsen 1977; Nulsen et al. 1982; Stewart et al. 1984; Fabian 1994; Tamura et al. 2001; Cavagnolo et al. 2009). However, this cooling is neither accompanied by strong star formation nor a significant fraction of gas colder than $1 \mathrm{keV}$ (e.g., Peterson et al. 2001; Rafferty et al. 2006; McNamara \& Nulsen 2007). Instead galaxy formation is halted by an unknown energy source (e.g., Croton et al. 2006).

A prominent theory is that energetic feedback from active galactic nuclei (AGNs) is required to explain these two discrepancies (e.g., Scannapieco \& Oh 2004; Springel et al. 2005; Dunn \& Fabian 2006; Thacker et al. 2006b; Sijacki et al. 2007; Booth \& Schaye 2009; Dubois et al. 2013; Martizzi et al. 2013). AGNs are among the most energetic objects in the universe, characterized by their extremely luminous cores powered by the infall of gas from a relativistic accretion disk (e.g., Rees 1984) onto a supermassive black hole (SMBH) with a mass $M_{\mathrm{BH}}>10^{6} M_{\odot}$. AGNs are associated with two modes of feedback into their environments. The kinetic or radio mode is associated with collimated relativistic jets, and low and radiatively inefficient accretion rates (e.g., Falcke \& Biermann 
1999; Sambruna et al. 2000; Merloni \& Heinz 2007), while the quasar or wind mode is associated with isotropic energy deposit and high and radiatively efficient accretion (e.g., Silk \& Rees 1998). Depending on the feedback mode, it has been shown that AGNs can provide the energy needed to maintain the hot ICM (e.g., Dunn \& Fabian 2006), with kinetic feedback creating the large buoyant bubbles (e.g., Dunn et al. 2006). It is also clear that this feedback can hamper cooling of galactic halo gas, preferentially reducing the SFR first in large halos at early times and then smaller halos at late times (e.g., Scannapieco \& Oh 2004; Scannapieco et al. 2005). AGNs also act to remove, via accretion and heating, the low specific angular momentum (sAM) gas from the central region of its halo, gas that would otherwise form stars. This leads to a net increase in the galactic sAM. However, AGNs can also decrease galactic sAM by heating the surrounding high angular momentum gas and preventing its accretion into the galactic disk (e.g., Dubois et al. 2013; Genel et al. 2015; Nelson et al. 2015).

Unfortunately, AGN feedback is extremely difficult to simulate, as its effects span several orders of magnitude, originating on sub-parsec scales and impacting kiloparsec and even megaparsec scales. Thus numerical methods must implement a subgrid prescription for injecting the feedback model if they are resolving cluster or cosmological scales. The nature of this feedback is also highly debated, with different studies focusing on different input mechanisms for the feedback energy and different environments in which to study its effects.

The first models of AGN feedback were limited to smoothed particle hydrodynamics (SPH) simulations. Springel et al. (2005) used the SPH code GADGET-2 (Springel 2005) to study individual and merging galaxies with black holes (BHs) and feedback injected into the simulation thermally and isotropically. They found that the feedback energy was sufficient to regulate the $\mathrm{BH}$ growth. Thacker et al. (2006b, 2009) carried out SPH simulations using the HYDRA (Couchman et al. 1995; Thacker \& Couchman 2006) code with an isotropic kinetic outflow model for AGN feedback. Their results reproduced the antihierarchical turnoff in the quasar luminosity function as well as the spatial distribution of quasars on both small and large scales. However, the impact of feedback was significantly less than predicted by analogous semianalytic models, a difference that could be traced to in-shock cooling as it occurred in the SPH simulation. Sijacki et al. (2007) introduced a dual mode prescription, accounting for both low-accretion rate radio, or kinetic, mode and high-accretion rate quasar, or thermal, mode feedback, for their AGN simulations in GADGET 2. This resulted in better agreement between the simulated galaxy stellar mass density and observations than previous models. Booth \& Schaye (2009) performed SPH simulations using the GADGET 3 code with a modified version of the AGN prescription of Springel et al. (2005) and found that the BHs greatly suppressed the star formation in high-mass galaxies, self-regulating their feedback such that they agreed with the $M_{\mathrm{BH}}-\sigma_{\mathrm{v}}$ relation, a tight correlation between $\mathrm{BH}$ mass, $M_{\mathrm{BH}}$, and the velocity dispersion of the host galaxy's bulge, $\sigma_{\mathrm{v}}$ (e.g., Ferrarese \& Merritt 2000; Gebhardt et al. 2000; Tremaine et al. 2002). Recently, Planelles et al. (2014), Le Brun et al. (2014), and Pike et al. (2014) used variants of GADGET 3 and GADGET 2 to study the impact of AGNs on a range of galaxy masses and clusters, and how AGN models can be tuned to produce better agreement between simulated and observed galaxy groups and clusters. Barai et al. (2013) and Wurster \& Thacker (2013) each used SPH simulations (GADGET 3and HYDRA, respectively) to consider the impact of various AGN feedback models on isolated galaxies and mergers, showing some success in replicating the $M_{\mathrm{BH}}-\sigma_{\mathrm{v}}$ relation.

All such SPH simulations, which use Lagrangian schemes to solve the equations of fluid dynamics, are very efficient at resolving dense structures. However, SPH is not without its shortcomings. As particles move along with the mass, SPH is ill-equipped to resolve the low-density environment surrounding galaxies and clusters, although it is through this medium that the feedback interacts with the surrounding structure. Furthermore, traditional, also called standard, SPH has difficulties accurately modeling shocks and mixing (e.g., Morris 1996; Marri \& White 2003; Agertz et al. 2007; Hopkins 2013), which are essential when studying the impact of feedback on surrounding structure. Fortunately, there is ongoing effort and success in reformulating the SPH method to overcome the mixing and shock issues discussed above (e.g., Price 2012; Hopkins 2013), although they have not yet become the standard practice.

Thus, recently, adaptive mesh refinement (AMR) methods have been gaining interest. As AMR is a shock-capturing method, it can set high spatial resolution in any region of the simulation volume and resolves shocks with only a few cells. Dubois et al. (2010) introduced kinetic radio-mode feedback in RAMSES simulations, and then in Dubois et al. (2013) used both radio and quasar models of AGN feedback in the AMR code RAMSES (Teyssier 2002) to study the growth of a galaxy cluster in a full cosmological simulation. Their study focused on the accretion history of the cluster SMBH and the effect of feedback on the gas content and temperature. They found that only with AGN feedback were they able to greatly heat the gas, and affect its ability to accrete onto the central galaxy, thus limiting the overall SFR and accretion on to the SMBH, while being in good agreement with the empirical $M_{\mathrm{BH}}-\sigma_{\mathrm{v}}$ relation. Martizzi et al. (2013) also used RAMSES to simulate an isolated cluster halo, studying how quickly cluster gas heated by AGN feedback cools back into the central region. Finally, Sembolini et al. (2016a) used RAMSES as well as a combination of SPH and moving-mesh simulation codes to better understand the impact of the numerical method on modeling the role of AGNs in galaxy cluster evolution. Consistent across their simulations, they find that the inclusion of AGN feedback prevented overcooling in their cluster core, leading to a stellar component more consistent with observations and increasing the fraction of gas at all radii.

Aside from using different hydrodynamical methods, these studies are quite diverse in how they model the deposited feedback energy and its impact on the environment. Thus the role of the simulation method in determining the conclusions of these studies is difficult to disentangle in the absence of a comparison that employs the same physical model across different simulation codes. The importance of comparisons between simulation techniques has also been highlighted by the usefulness of such studies in models of structure formation without AGN feedback. For example, the Santa Barbara Cluster Comparison Project (Frenk et al. 1999) compared the results of 12 numerical codes using the same initial conditions to study the virialization of a massive galaxy cluster without including feedback or radiative cooling, finding that agreement 
was best for properties of the dark matter and worst for the total X-ray luminosity. Similar comparisons with higher resolution were done in Voit et al. (2005) and then Mitchell et al. (2009), focusing on the central entropy profiles of clusters, and they showed that the core entropy was lower in SPH than in AMR due to under-mixing in SPH, and to a lesser degree, overmixing in AMR. A detailed computational comparison between AMR and SPH methods for standard hydrodynamic turbulence was performed by Agertz et al. (2007), which demonstrated the inherent difficulties of modeling shear layers with standard SPH. This work also highlighted the effect of steep density gradients in standard SPH simulations, where an effective surface tension between the two phases would lead to limited mixing, overcooling, and angular momentum transport (Kaufmann et al. 2007).

Code comparisons continue to be important. For the Aquila project, Scannapieco et al. (2012) studied the formation of a Milky-Way-sized galaxy, wherein 13 different numerical methods were used, starting from the same initial conditions, with no attempt to use identical sub-grid models (gas cooling and the formation and feedback of stars and AGNs). They found that while the variety of different gas physics led to a large span in the physical characteristics of the final galaxy, it was inconsistent with observations of real galaxies. They also found that gas cooled more efficiently in grid codes, leading to higher SFRs. In contrast to the Aquila project, the now underway AGORA project is combining the works of 95 scientists to use a variety of numerical codes with as identical as possible implementations of various baryonic physics to produce more observationally consistent galaxies (Kim et al. 2014). There has been a suite of work comparing galactic and cosmological simulations from the recently introduced movingmesh code AREPO, with the SPH code GADGET 3 (e.g., Springel 2010; Bauer \& Springel 2012; Kereš et al. 2012; Sijacki et al. 2012; Torrey et al. 2012; Vogelsberger et al. 2012; Nelson et al. 2013), which only recently included AGN feedback (Hayward et al. 2014). These have further demonstrated where shortcomings exist in the ability of SPH codes to mix merging material. In particular, Kereš et al. (2012) found that with radiative gas cooling, AREPO had either the same or lower central entropy profiles depending on the halo mass, which is the opposite of the results of grid codes in nonradiative simulations (e.g., Voit et al. 2005; Mitchell et al. 2009). Finally, the nIFTy Cosmology workshop has also lead to simulation comparison papers that include AMR and SPH codes, and the moving mesh code AREPO (Sembolini et al. 2016a, 2016b). For non-radiative simulations they reproduced the radial profile results of Mitchell et al. (2009). By introducing cooling and AGN feedback, at $z=0$ they see better qualitative agreement in the halo profiles, but with larger scatter.

In this work, we wish to continue the effort to compare the results from different codes as they simulate the cosmologically consistent formation of a cluster environment, including AGN feedback. We perform two simulation suites, one with AMR and one with standard SPH, from the same initial conditions, studying the impact of different subgrid physics, including cooling, star formation and stellar and AGN feedback models. Note that we attempt to implement nearly identical sub-grid baryonic physics models, using the same parameter values in these models to emphasize the role of the numerical method. We compare the ability of these two numerical methods to model the evolution of the cluster environment and its response to these subgrid models, including the gas temperature, SFRs, and gas content. We stress that these results are only applicable to standard SPH implementations, and future work comparing them with non-standard SPH implementations is required. Similarly, even where the two methods give consistent results, we emphasize that these predictions are limited to the single set of sub-grid models implemented in this work. In a companion paper (M. L. A. Richardson et al. 2016, in preparation) we compare the AMR and SPH impact of AGN feedback on the characteristics of halos ranging from $10^{11}$ to $10^{13.5} M_{\odot}$.

The structure of this paper is as follows. In Section 2 we discuss the cooling, star formation, and stellar feedback as well as AGN formation, accretion, merging, and feedback methods in both the grid and particle codes. In Section 3 we first give a detailed comparison of the results from our non-cooling and our fiducial simulations, where no AGN feedback is included. These constitute the backbone of our analysis, as it is only with respect to these non-AGN runs that we can determine how AGNs affect the cluster growth in AMR and SPH simulations. We then present the results from our simulations with AGN feedback. We give a discussion and conclude in Section 4.

\section{NUMERICAL METHODS}

Simulations were conducted with either the AMR code RAMSES (Teyssier 2002) or the SPH code HYDRA (Couchman et al. 1995). The initial conditions were generated using the mpgrafic (Prunet et al. 2008) package which creates a realization of the density fluctuations on a grid according to the desired power spectrum, and uses the Zel'dovich approximation to calculate the corresponding particle velocities. Our initial conditions were generated at a redshift of $z=43.2$, centered on a region in which a cluster halo with virial mass $M_{\text {vir }}=2 \times 10^{15} M_{\odot}$ forms by $z=0$. We assumed a $\Lambda$ CDM cosmology with cosmological parameters $\left(\Omega_{\Delta}, \Omega_{\mathrm{M}}, \Omega_{\mathrm{b}}, \sigma_{8}\right.$, $h)=(0.73,0.27,0.044,0.8,0.7)$ from the seven- year WMAP (Komatsu et al. 2011). Our simulations were carried out in a $100 \mathrm{~h}^{-1} \mathrm{Mpc}$ comoving box with periodic boundaries and were run to $z=3$. In both particle and grid simulations we assumed a zoomed-in realization of this box with a spherical highresolution region $25 \mathrm{~h}^{-1} \mathrm{Mpc}$ in diameter, an effective dark matter particle number of $1024^{3}$, and a mass resolution of $8.3 \times 10^{7} M_{\odot}$. This high-resolution region was selected to contain all particles found in the halo by $z=1$, and thus constitutes a conservative estimate of the necessary highresolution region for $z=3$. Outside of the high-resolution region, we uniformly decreased the dark matter particle resolution until reaching an effective particle number of $64^{3}$ for AMR and $128^{3}$ for SPH in the outer regions of the box. The dark matter particle initial conditions were identical between the grid and particle simulations.

We carried out the grid simulations with the RAMSES code (Teyssier 2002), which uses an unsplit second-order Godunov scheme to evolve the Euler equations for the gas. RAMSES variables are cell-centered and interpolated to the cell faces for flux calculations, which are then used with a Harten-Lax-van Leer-Contact Riemann solver (van Leer 1979; Einfeldt 1988). To calculate the gravitational potential and acceleration, the masses of a collisionless star, $\mathrm{BH}$, and dark matter particles were mapped to the grid with a cloud-in-cell (CIC) scheme (Birdsall \& Fuss 1997). The CIC method was also used to compare gas and particle densities for star formation and sink 
particle generation as discussed further below. Gas within the high-resolution region was refined using a semi-Lagrangian technique. When more than eight dark matter particles were in a cell, or when the baryon density in a cell was eight times more than the cosmic average, the cell was split into eight, doubling the spatial resolution. We aimed for a fixed maximum physical resolution of $\Delta x_{\min }=545 \mathrm{pc}$, where the maximum refinement level was increased with increasing cosmic scale factor, with increments occurring at $z \simeq 39,19,9$, and 4 . Thus the spatial resolution at any one time varied from 435 physical pc to 870 physical pc. To avoid over-resolving the dark matter in dense gas regions, we set the maximum level to map the dark matter particles into cells at $l_{\text {max,DM }}=15$. For comparison, at $z=4$, the gas was refined up to $l_{\text {max, }}=16$ levels of resolution. Thus from $z=4-3$ the dark matter had an effective softening length of 2 physical kpc, given by twice the width of a grid cell at $l=15$.

We carried out the particle-based simulations with the HYDRA code (Couchman et al. 1995; Thacker \& Couchman 2006), which uses an adaptive particle-particle, particle-mesh method (Couchman 1991) to calculate the gravitational forces and an SPH method (Gingold \& Monaghan 1977; Lucy 1977) to calculate the hydrodynamic forces. This is a standard implementation of SPH without a forced conservation of entropy (e.g., Springel 2005) and does not include methods for better capturing contact discontinuities (e.g., Hopkins 2013). We used the same initial conditions for dark matter as for the RAMSES run and overlaid the gas particles onto the dark matter particle positions, which then trace the gas density field. Note that HYDRA employs a modification of the kernel gradient to prevent the formation of the pair instability (Thomas \& Couchman 1992), and where pressure is important we confirm that gas and DM particles separate within a few time steps. HYDRA uses the S2 gravitational softening length (Hockney \& Eastwood 1981), $\epsilon$, for mediating close encounter scattering events, with the minimum smoothing length given by $h_{\text {min }}=\epsilon / 2$. We set $h_{\min }=2 \Delta x_{\min }=1090$ physical pc, giving the same softening length for dark matter particles in the two codes at late redshifts. Although the spatial resolution of a grid or particle method is not exactly equal to these two quantities, we found that the star formation histories were sufficiently similar when relating these parameters in this way. We discuss this further in Section 2.1. Each gas particle set its smoothing length such that it overlapped with roughly 52 neighbors, although at any given step this number could vary between 32 and 82 .

Optically thin, atomic cooling from hydrogen, helium, and metals was calculated following Sutherland \& Dopita (1993) for temperatures above $10^{4} \mathrm{~K}$, and for metal fine-structure cooling following Rosen \& Bregman (1995) at cooler temperatures. The gas temperature was not allowed to drop below $T_{0}=500 \mathrm{~K}$ via radiative and metal line cooling, although adiabatic cooling below this limit was permitted. After $z=8.5$, heating from an ultraviolet background was modeled following Haardt \& Madau (1996). For simplicity and consistency between codes, the metallicity was set at a constant value of a third solar for the entirety of the simulation, as typically observed in the intracluster medium (Loewenstein 2004). We note that a recently identified error in the cooling prescription in RAMSES shows that its metal cooling tables also include the contribution from hydrogen and helium, thus in regions where $\mathrm{H}$ and $\mathrm{He}$ are the main source of cooling (e.g., high temperature synchrotron tail) we were adding an extra third of cooling (since this extra term scales with the metallicity). However, for consistency, the same cooling tables were used in the HYDRA simulation, thus this error does not account for any differences between the two methods.

\subsection{Star Formation and Stellar Feedback}

In both types of simulations, star formation followed a Schmidt-Kennicut law (Schmidt 1959; Kennicut 1998), with the SFR given as

$$
\frac{d M_{*}}{d t}=c_{\mathrm{sf}} \frac{M_{\mathrm{g}}}{t_{\mathrm{ff}}},
$$

where $c_{\mathrm{sf}}$ is the star-forming efficiency, $M_{\mathrm{g}}$ is the gas mass, and $t_{\mathrm{ff}} \equiv(32 G \rho / 3 \pi)^{1 / 2}$ is the gravitational free-fall time in the vicinity of the star-forming region, with $G$ the gravitational constant and $\rho$ the gas mass density in a given resolution element. We set $c_{\mathrm{sf}}=0.01$, consistent with observations of giant molecular clouds (Krumholz \& Tan 2007). Star formation was implemented where the gas density was above a hydrogen number density threshold, sufficient to overcome the local hydrodynamic pressure. For these resolutions, we set this threshold to be $n_{*}=0.05$ hydrogen atoms $\mathrm{cm}^{-3}$. We also incorporate a baryon overdensity threshold, comparing the local density of gas and stars to dark matter. This is necessary to prevent star formation from proceeding in cosmic filaments at high redshift. For SPH, if the total baryon mass within two smoothing lengths of a gas particle was more than 0.25 that of the enclosed dark matter, then the particle was permitted to proceed with the star formation prescription discussed below. For AMR, we checked on a cell-by-cell case and required a stricter threshold than SPH to ensure the gas in the smaller volume was indeed dominating the local mass. We allowed this to vary with time since the dark matter softening transitioned to $1 \mathrm{kpc}$, and ensured agreement with the global SFR in the SPH simulation in the zoom-region of the box. This resulted in the gas overdensity threshold ranging from 0.25 to 20 times the dark matter mass in a given cell. We assumed that cold gas above the density threshold belonged to the multiphase interstellar medium (ISM), which we could not resolve. As such we employed a polytropic equation of state for such gas with $T=T_{0}\left(n / n_{*}\right)^{\kappa-1}$, with a polytropic index $\kappa=4 / 3$ and an ISM temperature $T_{0}=500 \mathrm{~K}$. The density is also consistent with that of particle simulations of similar resolution (e.g., McCarthy et al. 2010; Scannapieco et al. 2012 and references therein; Sijacki et al. 2012; Hayward et al. 2014).

The implementation of star formation in RAMSES is described in Rasera \& Teyssier (2006). We defined a unit stellar mass, $m_{*, R}=\Delta x_{\min }^{3} n_{*} m_{\mathrm{p}} / X_{\mathrm{H}}$, where $m_{\mathrm{p}}$ is the proton mass and $X_{\mathrm{H}}=0.76$ is the hydrogen mass fraction. Thus the unit stellar mass is the mass in a cell at the threshold density, and is therefore the minimum stellar mass. If a cell's gas density was above the star formation threshold density and overdensity then we used Equation (1) with $M_{\mathrm{g}}=\Delta x^{3} \rho$ to determine the amount of stellar mass expected to be created in the next time step. Comparing this expected mass with the unit stellar mass yielded an expected number of stars to be formed, which was used as the expectation value for a random integer drawn from a Poisson distribution. A star particle was then 


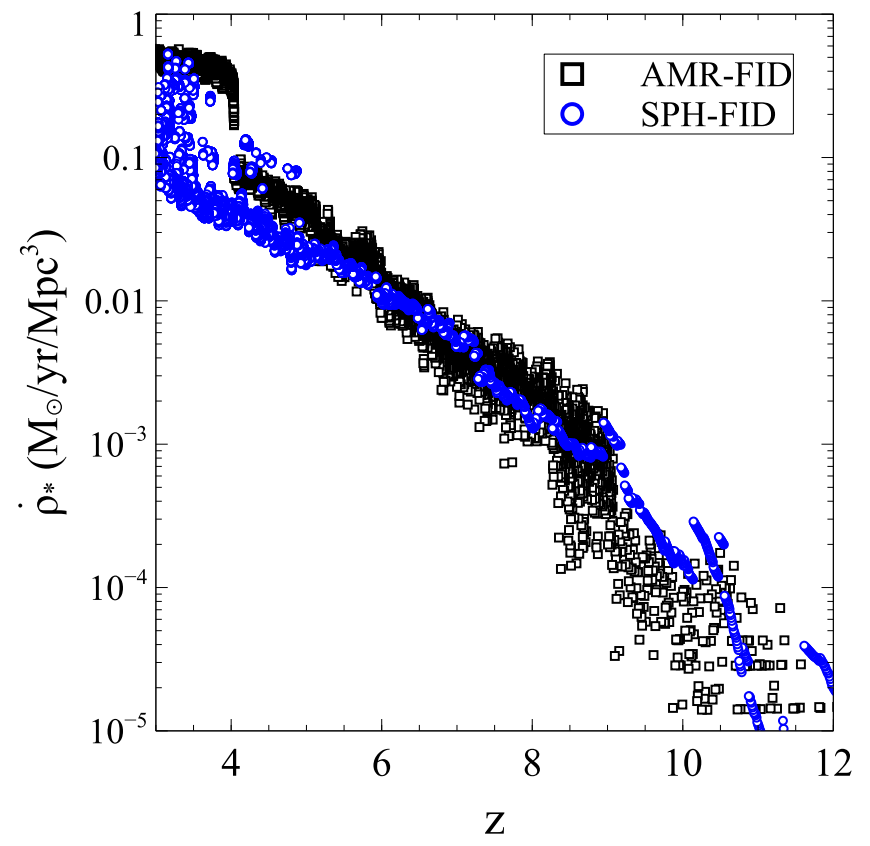

Figure 1. Star formation density history up to $z=3$ in the zoom region, comparing HYDRA and RAMSES. Black squares show the global star formation rate density in the high-resolution zoom region for RAMSES while blue circles show the results for HYDRA. Note the delayed star formation for RAMSES runs near $z=4$. The maximum refinement level increases at this redshift, followed by a strong increase in star formation as we better resolve the central density peak of star-forming clumps. The best agreement is above $z \approx 5$ and at $z \approx 3$, when the resolutions were most comparable.

generated with the same velocity as the cell and a mass equal to the random number times the unit stellar mass, and the cell's mass was reduced by the star particle's mass.

The implementation of star formation in HYDRA is described in detail in Thacker \& Couchman (2000). Each gas particle accumulated a stellar component following Equation (1) where $M$ was that gas particle's mass and $\rho$ was the local gas density at the particle. Once the accumulated mass was equal to the star particle mass, which we set to be half the high-resolution gas particle mass, a star particle was formed, and the gas particle's mass was equivalently reduced. Until the time at which a star was formed, the gas dynamics used the total gas particle mass, not just the non-stellar component. The only exception to this was that the mass in Equation (1) only included the non-stellar component of the gas particle. Once a gas particle that already made one star particle had converted $80 \%$ of its remaining mass into stars, then that entire gas particle was turned into a second star particle.

In Figure 1 we compare the history of star formation for the two codes in a $25 h^{-1}$ comoving Mpc sphere centered on the region of interest. The varying resolution of the RAMSES runs is manifested by the delay in star formation shortly before an increase in maximum refinement level, occurring at roughly $z=9$ and $z=4$. While for $h_{\min }=2 \Delta x$ there was fairly good agreement before using the overdensity threshold discussed above, the inclusion of this threshold results in stars forming only in galaxy cores.

We assumed $10 \%$ of the stellar mass formed was contained in high-mass stars that contributed feedback through type II supernovae (SNe), with $10^{51} \mathrm{erg}$ of energy released for $10 M_{\odot}$ of high-mass stars (i.e., per $100 M_{\odot}$ of total stars). We thus used a total efficiency of $\epsilon_{\mathrm{fb}}=5 \times 10^{15} \mathrm{erg} \mathrm{g}^{-1}$ (Sommer-Larsen et al. 1999). This energy was deposited immediately into the vicinity of the formed star particle. Although the typical lifetime of a high-mass star was on the order of tens of timesteps, because our single particle is representative of several giant molecular clouds and our resolution is on the order of kiloparsecs we argue that injecting this energy without a delay will not change the nature of subsequent star formation compared with a model that accounts for this delay (see Wurster \& Thacker 2013). The implementation of the stellar feedback in RAMSES is described in Dubois \& Teyssier (2008). This energy was deposited kinematically with a radius of a single cell around the star particle. The injected mass, momentum, and energy are consistent with a Sedov blast wave solution. The implementation of the stellar feedback in HYDRA is described in Thacker \& Couchman (2000). Since particle positions are not isotropic, we injected this energy thermally, using a kernel weighting for gas particles within the star particle's former smoothing length. Post-feedback cooling uses a multiphase description of the gas density, where the heated gas' density is much lower than that of the cold gas. In this model, pressure equilibrium is assumed, and the resulting decrease in density is determined by the net energy increase. In this way, the method is similar to other delayed cooling schemes (see, for example, Gerritsen \& Icke 1997). This multiphase density then gradually returns to the particle density, set by a half-life time of $1 \mathrm{Myr}$, consistent with the time for the blast wave to reach the cooling radius for densities near our star formation threshold and feedback energy (Blondin et al. 1998).

\subsection{BHs and AGN Feedback}

BHs were modeled as sink particles in both types of simulations, and they were formed where the local gas and stellar density were both above the star formation criteria, $n_{*}=0.05 \mathrm{~cm}^{-3}$. All BHs had a seed mass of $8 \times 10^{5} M_{\odot}$, and to ensure only one $\mathrm{BH}$ was made per galaxy, they were only allowed to form in locations at least 30 comoving $\mathrm{kpc}$ from all other BHs. In RAMSES, this particle was given the same momentum as its cell of origin, and the cell's mass was reduced by the seed mass. In HYDRA, the BHs were spawned at the same location and with the same momentum as the source gas particle, and the gas mass was reduced by the seed mass. Each BH particle in HYDRA had a smoothing length that overlapped with roughly 60 neighboring gas particles. BHs could merge when they were within four resolution units in AMR or two smoothing lengths in SPH.

The BHs accreted gas following the Bondi-Hoyle-Littleton rate (Bondi 1952), as described in Dubois et al. (2013) and Wurster \& Thacker (2013). The accretion rate is given by

$$
\frac{d M_{\mathrm{BH}}}{d t}=4 \pi \alpha \frac{G^{2} M_{\mathrm{BH}}^{2} \bar{\rho}}{\left(\bar{c}_{\mathrm{s}}^{2}+\bar{u}^{2}\right)^{3 / 2}},
$$

where $M_{\mathrm{BH}}$ is the $\mathrm{BH}$ mass, $\bar{\rho}$ is the local average gas density, $\bar{c}_{\mathrm{s}}$ is the local average sound speed, $\bar{u}$ is the local average gas speed, and $\alpha$ is a dimensionless boost factor with $\alpha=\max \left[1,\left(n / n_{*}\right)^{2}\right]$ (Booth \& Schaye 2009), which accounts for our inability to resolve the cold, high-density ISM gas around the $\mathrm{BH}$. We set the maximum accretion rate to be the 
Eddington accretion rate,

$$
\frac{d M_{\mathrm{Edd}}}{d t}=4 \pi \frac{G M_{\mathrm{BH}} m_{\mathrm{p}}}{\epsilon_{\mathrm{r}} \sigma_{\mathrm{t}} c},
$$

where $\sigma_{\mathrm{t}}$ is the Thompson cross section, $c$ is the speed of light, and $\epsilon_{\mathrm{r}}$ is the radiative efficiency, set to 0.1 for the Shakura \& Sunyaev (1973) model of accretion onto a Schwarzschild BH. In RAMSES, each $\mathrm{BH}$ particle has a cloud of sensors that samples the surrounding gas to determine the average gas quantities. In HYDRA we used a kernel-weighted average of the gas particles that overlap with the BH's smoothing length. Since mass is discretized in HYDRA, we used an internal and a dynamic BH mass. The internal mass was incremented by the accreted amount, and once this mass was larger than the dynamical mass by half a gas particle mass, the closest gas particle was accreted and the dynamical mass was increased by this amount. This dynamical mass was used to calculate the gravitational effect of the $\mathrm{BH}$, while the internal mass was used to determine accretion and feedback properties.

The $\mathrm{BH}$ particles were advected similar to the dark matter particles. To model the effect of gas on the BHs, we included a drag force, thus avoiding spurious oscillations of the BHs about their local potential minimum. This dynamical friction is set to $F_{\mathrm{DF}}=f_{\text {gas }} 4 \pi \alpha \rho\left(G M_{\mathrm{BH}} / \bar{c}_{\mathrm{s}}\right)^{2}$, where $f_{\text {gas }}$ is a factor whose value depends on the local Mach number, with $0<f_{\text {gas }}<2$ (Ostriker 1999; Chapon et al. 2013). In HYDRA, the drag force is calculated using the smoothed gas values of the BH's neighboring particles.

In this work, we have employed the quasar-mode AGN feedback following Dubois et al. (2013). At every step the thermal quasar feedback mode injected energy into a sphere of radius $\Delta x$ centered on the $\mathrm{BH}$, at an injection rate of $\dot{E}_{\mathrm{AGN}}=\epsilon_{\mathrm{f}} \epsilon_{\mathrm{r}} \dot{M}_{\mathrm{BH}} c^{2}$, where $\epsilon_{\mathrm{f}}$ is a free parameter set to 0.15 to reproduce the $M_{\mathrm{BH}}-M_{\mathrm{b}}, M_{\mathrm{BH}}-\sigma_{\mathrm{b}}$, and $\mathrm{BH}$ density in the local universe (see Dubois et al. 2012). This value is also consistent with observations of the kinetic power in quasar outflows, where a lower limit of a few percent of the bolometric luminosity is seen (e.g., Moe et al. 2009; Maiolino et al. 2012; Arav et al. 2013). The implementation of this feedback mode in HYDRA was built upon the existing work by Wurster \& Thacker (2013), which heats gas particles within two smoothing lengths. For both methods, the feedback was deposited with a uniform specific energy inside the bubble radius. Thus, our feedback model injects thermal energy isotropically. Anisotropic evolution of this heated gas is caused by its interaction with the surrounding clumpy structure. However, with this sub-grid model we cannot account for the clumpy, multiphase medium expected on scales corresponding to the accretion disk and Bondi radius of the AGN, which may well lead to anisotropic injection of the feedback energy. Future, larger simulations will be better placed to model this more compact region, although implementing an identical model in the two different simulation methods for consistent comparison may prove difficult.

For each simulation code, we ran three simulations (see Table 1). One simulation was carried out without cooling, reionization, star formation, or AGNs, so as to best understand, from the ground up, how the two methods compare. This simulation is labeled AMR-NC and SPH-NC (for no cooling) in RAMSES and HYDRA, respectively. A second simulation includes cooling, reionization, star formation, and $\mathrm{SNe}$ feedback. This simulation is labeled AMR-FID and SPHFID (for fiducial) in RAMSES and HYDRA, respectively. A final simulation includes these processes, but additionally tracks the evolution of AGNs and their associated feedback. This is labeled AMR-QSO and SPH-QSO in RAMSES and HYDRA, respectively. For the QSO run, to make the two implementations as similar as possible, we used a temperature ceiling of $10^{10} \mathrm{~K}$, and in the SPH run, energy was deposited kernelweighted to the gas particles within the $\mathrm{BH}$ smoothing length. Regardless of the simulation method, if the AGN feedback energy at a given step would heat its environment above the temperature ceiling, then the excess energy was saved for the following step and accretion was stalled until this excess energy was administered. With this work we wish to highlight the dependence of cluster environment and galaxy gas evolution on feedback implementations and how this evolution is dependent on the numerical method.

\section{RESULTS}

We break up our results into four main sections, as we focus on the cluster environment and its gas and how the cluster responds to the addition of increasingly complex gas physics in AMR and SPH simulations. We begin by looking at the characteristics of the gas in the cluster halo and sequentially increase the physics included in the simulations. A summary of the different simulations is given in Table 1 . We first discuss the non-cooling runs and then compare these simulations with our fiducial runs, which include radiative cooling, star formation, and stellar feedback. We then introduce our AGN simulations and compare these with our fiducial runs. For these first three sections we focus in great detail on snapshots at $z=5$ and $z=3$, and we end our results section by looking at the continuous evolution of the cluster gas, stellar, and $\mathrm{BH}$ components between these two snapshots.

\subsection{Non-cooling Runs}

We first ran the two NC simulations, which did not include gas cooling, star formation, reionization, or BHs. These two simulations are crucial for ensuring the gravity solvers are in good agreement and that the artificial viscosity implemented in HYDRA produces consistent shock heating. They also allow us to compare our simulations directly with other non-radiative work (e.g., Voit et al. 2005; Mitchell et al. 2009). Unlike the more complex cases, we only ran these simulations to $z=5$, which, given their simplicity, was sufficiently advanced to be able to compare them with the more complex runs. Virial quantities, taken at the radius within which the average density is 200 times denser than the critical density, are presented for all simulations in Table 2.

The left column of Figure 2 compares the density of the gas out to 8 virial radii surrounding the cluster at $z=5$ from the two simulations (top: AMR; bottom: SPH). Unsurprisingly, there is significant correspondence between the RAMSES and the HYDRA plots. However, we note significantly more dense gas present in the SPH run. We attribute this to a combination of the different gravitational calculations on small scales between the two methods and numerical dissipation of entropy in dense regions in SPH. On small scales HYDRA uses a particle-particle gravity solver, while RAMSES uses the standard adaptive particle mesh. Particle-particle methods have better short-range force resolution, which gives more 
Table 1

Simulation Summary

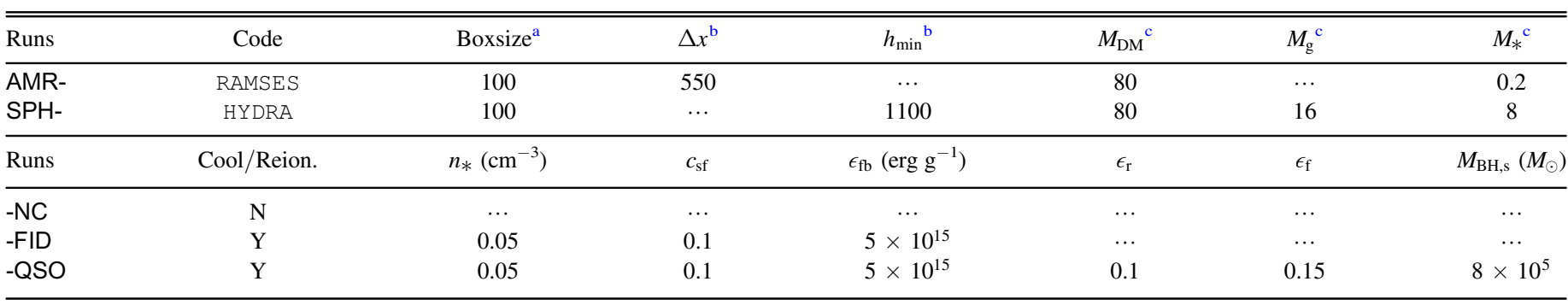

Notes.

${ }^{\text {a }}$ Comoving $\mathrm{Mpc} h^{-1}$.

${ }^{\mathrm{b}}$ Physical pc.

c ${ }^{3} 10^{6} M_{\odot}$.

Table 2

Cluster Characteristics

\begin{tabular}{|c|c|c|c|c|c|c|}
\hline$z$ & Run & $M_{\text {tot }}\left(M_{\odot}\right)$ & $r_{\mathrm{vir}}(\mathrm{kpc})$ & $T_{\text {vir }}(\mathrm{K})$ & $S_{\text {vir }}{ }^{\mathrm{a}}\left(\mathrm{keV} \mathrm{cm}{ }^{2}\right)$ & $\overline{\left(\frac{M_{\mathrm{bar}}}{M_{\mathrm{tot}}}\right) /\left(\frac{\Omega_{\mathrm{b}}}{\Omega_{\mathrm{m}}}\right)}$ \\
\hline \multirow[t]{6}{*}{5} & AMR-NC & $2.33 \times 10^{12}$ & 70.3 & $7.6 \times 10^{6}$ & 17 & 0.92 \\
\hline & AMR-FID & $2.30 \times 10^{12}$ & 70.0 & $7.5 \times 10^{6}$ & 17 & 1.1 \\
\hline & AMR-QSO & $1.95 \times 10^{12}$ & 66.2 & $6.7 \times 10^{6}$ & 15 & 0.68 \\
\hline & SPH-NC & $2.45 \times 10^{12}$ & 71.7 & $7.9 \times 10^{6}$ & 18 & 0.92 \\
\hline & SPH-FID & $2.54 \times 10^{12}$ & 72.5 & $8.0 \times 10^{6}$ & 18 & 0.92 \\
\hline & SPH-QSO & $2.26 \times 10^{12}$ & 69.9 & $7.4 \times 10^{6}$ & 17 & 0.68 \\
\hline \multirow[t]{4}{*}{4} & AMR-FID & $7.31 \times 10^{12}$ & 123.1 & $1.36 \times 10^{7}$ & 44 & 1.0 \\
\hline & AMR-QSO & $6.49 \times 10^{12}$ & 118.3 & $1.25 \times 10^{7}$ & 40 & 0.61 \\
\hline & SPH-FID & $7.27 \times 10^{12}$ & 123.6 & $1.35 \times 10^{7}$ & 44 & 0.92 \\
\hline & SPH-QSO & $6.77 \times 10^{12}$ & 120.5 & $1.29 \times 10^{7}$ & 41 & 0.68 \\
\hline \multirow[t]{4}{*}{3} & AMR-FID & $2.30 \times 10^{13}$ & 224.1 & $2.33 \times 10^{7}$ & 128 & 1.0 \\
\hline & AMR-QSO & $2.18 \times 10^{13}$ & 219.7 & $2.25 \times 10^{7}$ & 123 & 0.74 \\
\hline & SPH-FID & $2.31 \times 10^{13}$ & 227.3 & $2.30 \times 10^{7}$ & 126 & 0.92 \\
\hline & SPH-QSO & $2.19 \times 10^{13}$ & 223.2 & $2.25 \times 10^{7}$ & 123 & 0.68 \\
\hline
\end{tabular}

Note.

${ }^{a}$ Following Voit et al. (2005), but per $\mathrm{H}$ atom instead of electrons.

clumps (e.g., O'Shea et al. 2005). Additionally, HYDRA uses an energy-conserving rather than entropy-conserving implementation of SPH. Therefore, in dense regions some entropy is dissipated numerically, further differentiating these clumps in $\mathrm{SPH}$. The larger number of dense knots in classic SPH is well established, and has been discussed in detail (e.g., Frenk et al. 1999; Kaufmann et al. 2006; Power et al. 2014).

The middle and right columns of Figure 2 show the temperature in projection and slice, respectively, of the gas for the same region. The pixelation seen in the SPH slice is a result of the mapping used to produce the image with the same visualization software as for the AMR images (Turk et al. 2011, http://yt-project.org/). In projection we see better agreement between the two simulations, while the slices highlight the different extent of the virial shocks. The SPH run has a larger shock radius that is spread over a wider spatial extent, consistent with the artificial viscosity injecting entropy at a somewhat larger radii than in AMR. This earlier shock heating causes more high-entropy gas in SPH since the densities are very similar at the virial radius. This increased entropy may also be related to the poor ability of SPH to model subsonic turbulence, as described in Bauer \& Springel (2012). Gas accretion into clusters is dependent on subsonic gas, which in AMR correctly cascades to smaller scales but in
SPH thermalizes near the driving scale. It is this, combined with heating by the artificial viscosity, that leads to an expanded virial shock.

Figure 3 presents radial profiles of the gas density and entropy, here expressed as $S \equiv k_{\mathrm{B}} T / n_{\mathrm{H}}^{2 / 3}$, where $k_{\mathrm{B}}$ is the Boltzmann constant, and $n_{\mathrm{H}}$ is the hydrogen number density, for all gas, out to eight times the virial radius $\left(r_{\mathrm{vir}}=70\right.$ physical kpc). Virial quantities are annotated with dotted gray lines to aid in comparisons with works that look at multiple halos, switching to scale-free units normalized by these virial quantities (e.g., Voit et al. 2005; Mitchell et al. 2009; Kereš et al. 2012). Also included in the left plots of this figure are volume-averaged density and entropy (taken as the volumeaveraged temperature divided by the volume-averaged density to the $2 / 3$ power), which for direct comparison between AMR and $\mathrm{SPH}$, we normalize by the virial quantities.

For $0.2 r_{\text {vir }}<r<r_{\text {vir }}$, the average entropy and density profiles agree very well, and they are consistent with previous comparisons looking at a range of mass scales (Frenk et al. 1999; Voit et al. 2005; Mitchell et al. 2009; Richardson et al. 2013; Sembolini et al. 2016b). In particular, the entropy profile matches the observations of Voit et al. (2005), where $S \propto r^{1.2}$. The average AMR gas is slightly more dense through the virial shock, which may be due to SPH smoothing out the shock 


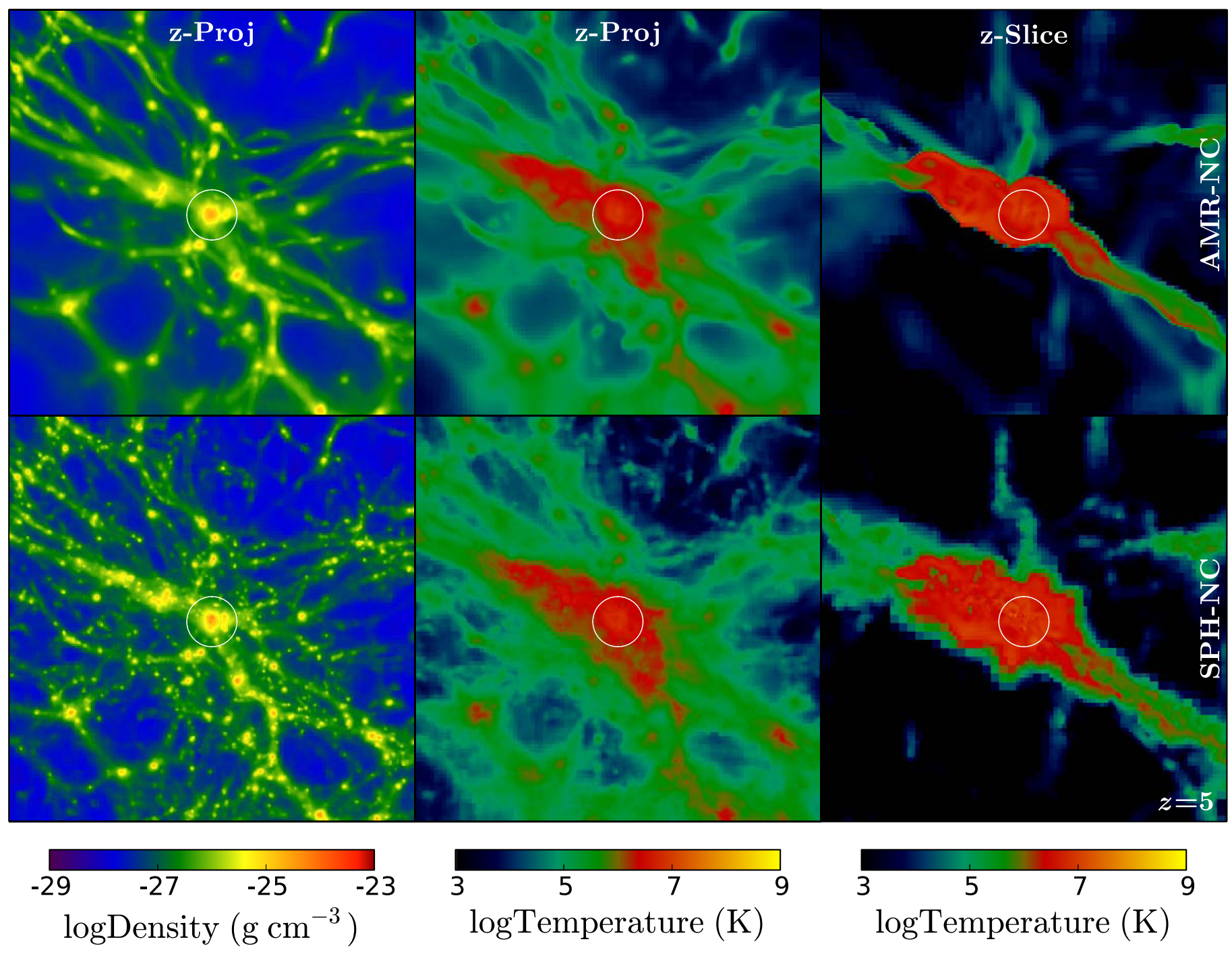

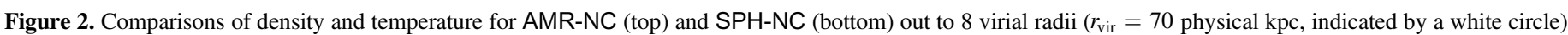

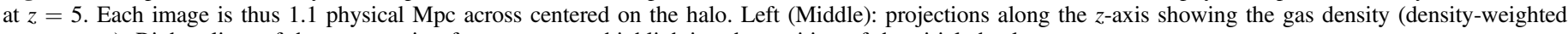
temperature). Right: slices of the same region for temperature highlighting the position of the virial shock.

width. Outside of the virial radius, the average density is again in agreement between the two methods, although the entropy is slightly increased in SPH near the virial radius. Sembolini et al. (2016b) saw a similar small increase in the entropy at the virial radius in their HYDRA simulation compared to their AMR comparison run. As we discussed above, this is due to higher temperature at the virial radius.

Within $0.2 r_{\text {vir }}$, on the other hand, the SPH gas density is higher than in AMR, and the entropy reaches a lower value than AMR before plateauing. The higher, more centrally peaked density in SPH is seen in Figure 2 in the center of the cluster. This is also consistent with previous comparisons (Frenk et al. 1999; Voit et al. 2005; Mitchell et al. 2009; Richardson et al. 2013; Sembolini et al. 2016b), and Mitchell et al. (2009) demonstrated that the lower entropy in the central region of SPH simulations is due to a reduced amount of mixing, with the low-entropy particles instead sinking to the center. It is unclear, however, just how much over-mixing occurs in AMR simulations, thus the true central profile is expected to be slightly lower than in AMR.
Looking at the full distribution of density beyond the virial radius, we see that the density in HYDRA extends to higher values than in RAMSES, consistent with satellite objects also collapsing to slightly higher densities in SPH, and visible in Figure 2. The entropy distribution is wider in the center of the SPH simulations, consistent with less mixing of high and low entropy particles.

Figure 4 shows two-dimensional entropy-density and temperature-density distribution functions for gas within the virial radius, along with one-dimensional distribution functions for each of these quantities. In the SPH simulation, highdensity gas is found at lower entropy and temperature and even higher densities than in the AMR case (compare with Figure 3). This gas is at the center of the cluster, where the entropy is numerically dissipated, and not accurately reinjected through mixing (Mitchell et al. 2009) or from large scales (Bauer \& Springel 2012). This larger fraction of high-density, lowentropy gas in SPH is also seen as halo substructure in the nIFTy comparison (Sembolini et al. 2016b). A larger fraction of gas is found at very high entropy in SPH, corresponding to low density, hotter gas at the virial radius, as we discussed 

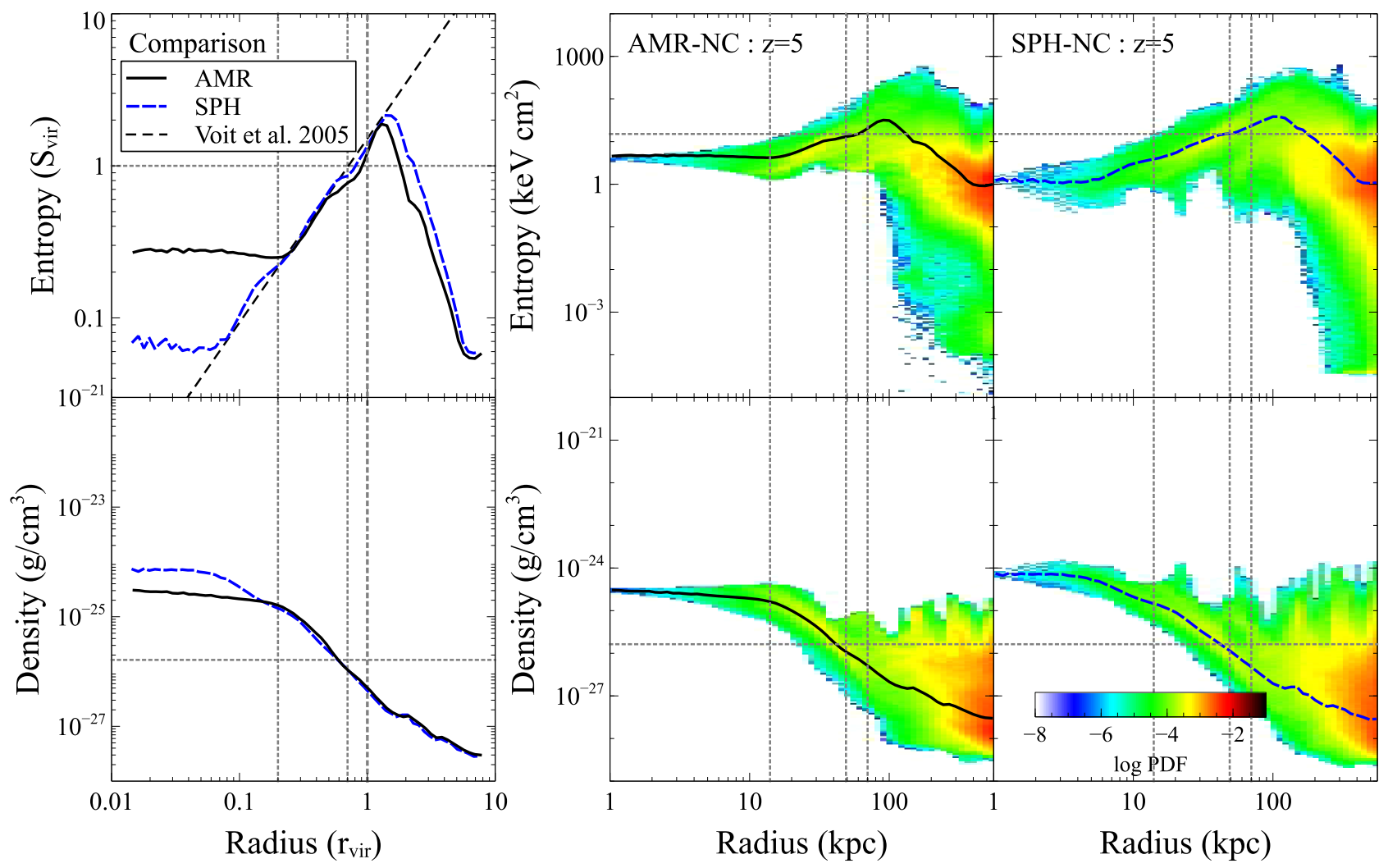

Figure 3. Radial profile plots at $z=5$ showing the gas entropy (top) and gas density (bottom) vs. radius for AMR-NC (middle) and SPH-NC (right) out to 8 virial radii from the cluster. 0.2, 0.7, and 1.0 virial radii, and the virial density and entropy are indicated by the gray dotted lines (see Table 2). The virial entropy is calculated following Voit et al. (2005), except we use the hydrogen density instead of the electron density. Color corresponds to log gas mass probability distribution fraction, where the integral of this quantity over a given plot is unity. The solid black (dashed blue) lines in the middle (right) plots demark the volume-weighted average density and entropy (taken as the average temperature over average density to the $2 / 3$ power) with radius for all gas in the AMR (SPH) simulation. The left plots compare the average values of AMR vs. SPH, using the same color and line scheme as the middle and right plots, scaled by the virial quantities so that they are directly comparable.

above. Besides the very high density gas in SPH, the distribution of density and temperature in SPH and AMR are very consistent.

In general, the subtle differences that appear between the simulations are not surprising due to the difficulty in implementing an ad hoc artificial viscosity in SPH, the energy-conserving implementation of the fluid equations in $\mathrm{SPH}$, and the tendency for SPH to undermix and AMR to overmix. We proceed, aware that these small differences may compound when including cooling, star formation, and feedback.

\subsection{Fiducial Runs}

Next we look at the FID simulations, which compare AMR and SPH with the inclusion of cooling, reionization, star formation, and stellar feedback. We first begin by comparing these results with the $z=5 \mathrm{NC}$ results, and then compare the FID results in more detail at $z=3$. The halo virial quantities are listed in Table 2, which shows that the inclusion of cooling has led to a higher gas fraction than in the AMR-NC simulations, with the AMR-FID values now slightly exceeding the cosmic average.

In Figure 5 we show projections of the gas density for the AMR-FID and SPH-FID simulations at $z=5$. In the FID simulations the gas can cool, resulting in more condensed structures. Thus the filaments are thinner, and the galaxies are collapsed to thin disks. On these scales in density we see little impact of star formation or feedback. In comparison, we see an amplification of the differences seen in Figure 2, with more clumps in SPH than in AMR. In AMR, the gas filaments are smooth ribbons of near uniform density gas with large galaxies residing within their nodes. In SPH, these filaments are much more inhomogeneous, housing many more small clumps that are less dense than AMR galaxies, but more dense than the surrounding filament gas. The clumpiness in SPH-NC is now compounded in SPH-FID by the fact that this denser, lowerentropy gas has shorter cooling times, leading to quicker fragmentation times for the filament as a whole. Inside the virial radius the dense gas is completely fragmented into individual parcels. The larger clumps agree between AMR and $\mathrm{SPH}$, and are cospatial with clumps in dark matter. However, the additional clumps found in SPH, which are seen in other studies of classic SPH (e.g., Frenk et al. 1999; Kaufmann et al. 2006; Power et al. 2014), are not cospatial with dark matter clumps and are due to artificial dissipation. We stress, however, that as the densest clumps fragment along the polytrope, they are forced to increase in temperature. Now that the filling factor of dense gas has decreased, we expect that the addition of AGN feedback will be more efficient in SPH and better able to blow away the more tenuous ambient gas, as we discuss in detail in Section 3.3.

In the fiducial runs the virial shocks in the SPH case are at significantly larger radii than those in the AMR case. These differences are visibile in the temperature projections and slices 


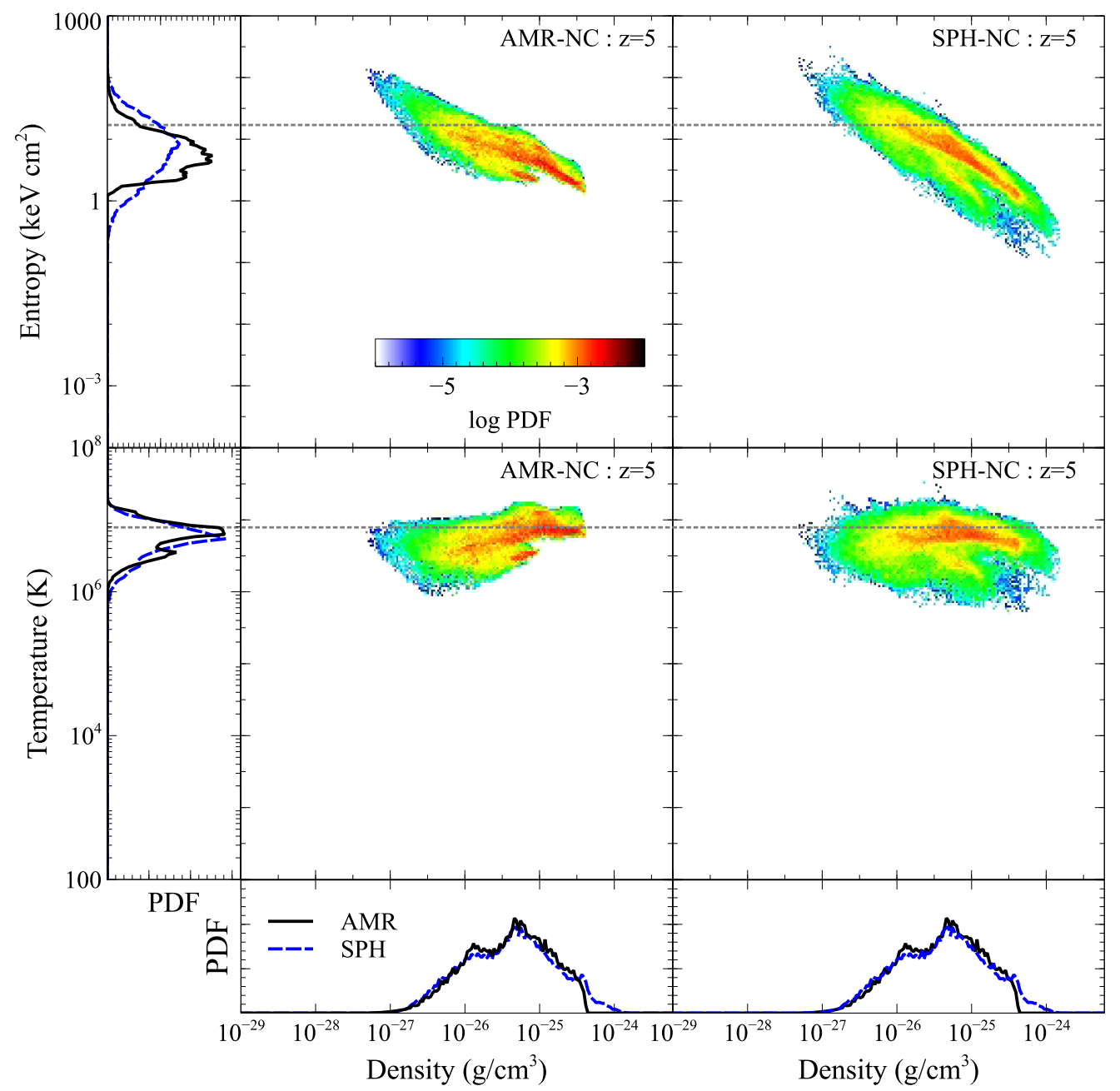

Figure 4. Phase plots at $z=5$ showing the gas entropy (top) and temperature (bottom) vs. density for AMR-NC (left) and SPH-NC (right) within the virial radius. Color corresponds to log gas mass fraction. The horizontal dotted line demarks the virial entropy and temperature of the cluster. On the left edge in linear units are onedimensional probability distributions of the log entropy (top) and log temperature (bottom) comparing the relative distribution of gas mass for both AMR (black solid line) and SPH (blue dashed line), while the bottom edge shows in linear units the one-dimensional probability distribution of log gas density, shown twice to facilitate comparison with the above phase plots.

shown in the middle and right panels of Figure 5 and are much more apparent than in the NC comparison runs shown in Figure 2. Thus the increased post-shock temperature and slightly wider shock in SPH (e.g., Hubber et al. 2013) leads to a lower cooling rate. We discuss the expected cooling times below.

Figure 6 presents the profile diagram of the gas at $z=5$ out to 8 virial radii, to compare with Figure 3 . Here we have split the average quantities into a hot component above $50,000 \mathrm{~K}$, and a total component. The hot gas is more directly relatable to other work (e.g., Kereš et al. 2012) and our NC runs and less susceptible to the different clumping behavior. Cooling in the fiducial case also leads to a large population of gas at very low entropies and high densities, which is seen in both the AMR and SPH simulations. However, in the AMR run there is a twophase medium, with cold and dense material found at the same radial distances as the hot, tenuous gas. In the SPH run, on the other hand, the hot and cold phases are segregated, with cold material found almost exclusively in the center, surrounded by a hot diffuse region. Thus, the addition of cooling has amplified the ability of low entropy gas in SPH to sink to the center of the halo, and the two methods yield very different results within $0.2 r_{\text {vir }}$.

Beyond this radius, out to $0.7 r_{\mathrm{vir}}$, there is better agreement of the hot gas between the two methods, while AMR is better able to model cold streams with an entropy of roughly $10^{-2} \mathrm{keV} \mathrm{cm}^{2}$. Beyond $0.7 r_{\mathrm{vir}}$ and out to roughly the virial radius, the entropy differences are even stronger than in the NC runs. Here the post-shock gas can efficiently cool in the AMR run, leading to the shock radius lying within the virial radius. In the SPH run, on the other hand, the shock radius is at the virial radius. This is not what is expected from theory. At $z=5$, this system has a dynamical time of roughly $0.11 \mathrm{H}^{-1}$, while the cooling time is approximately $0.03 \mathrm{H}^{-1}$. Thus the post-shock gas should cool quicker than the typical growth time of the halo, and we expect the virial shock to lie within the virial radius (e.g., White \& Rees 1978; Birnboim \& Dekel 2003). In our HYDRA run, no star particles have been made within the virial radius of this cluster by $z=5$, thus this larger virial shock and smaller filling factor of cold gas is not due to a difference in the SPH SNe feedback. Instead, this appears to be due to the excess heating near the virial radius seen in the SPH-NC run, coupled to a reduced cooling rate as the gas shock is broadened 

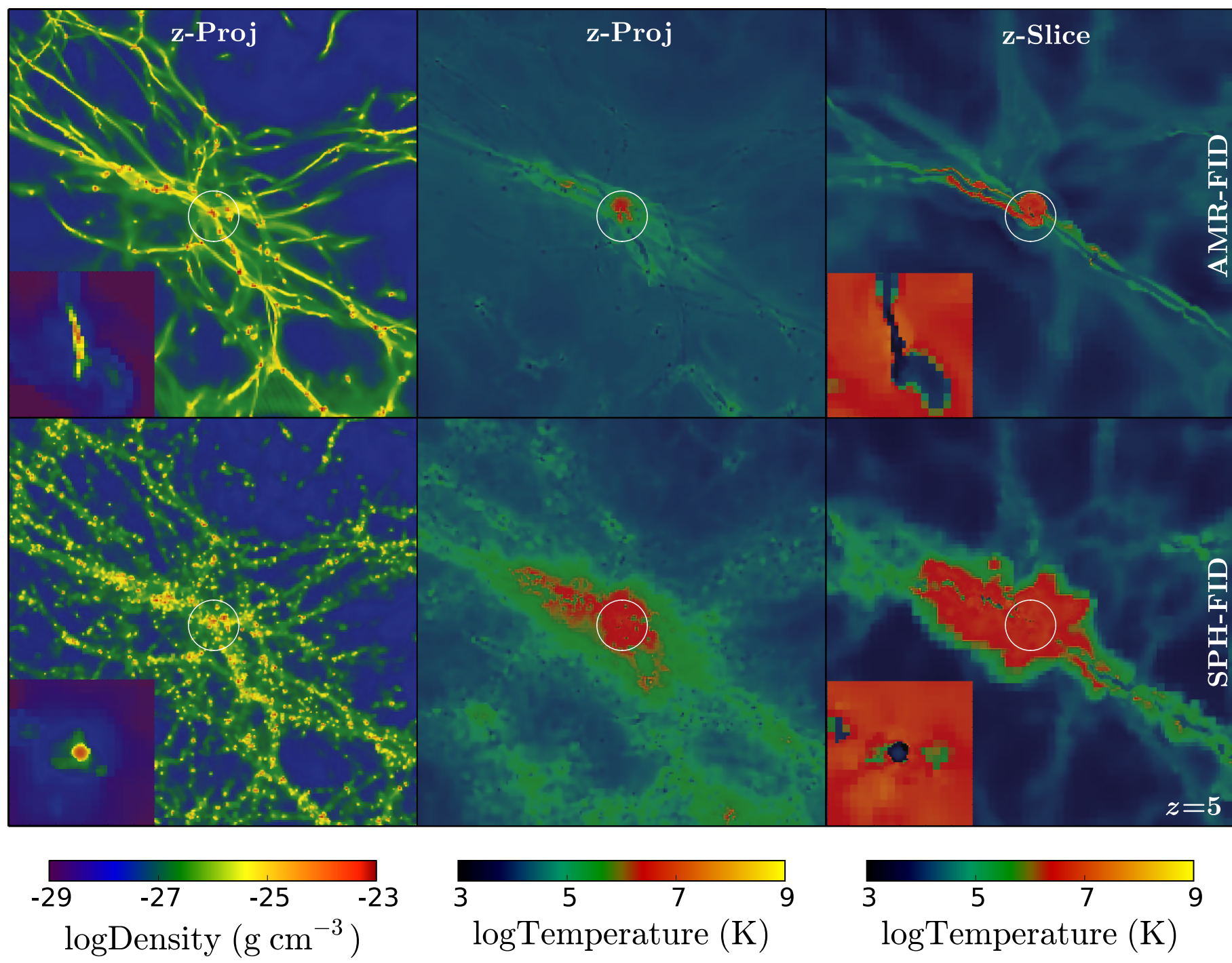

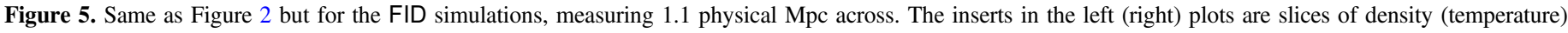
focused on the galaxy scale, measuring 28 physical kpc across, and reach out to $0.2 r_{\text {vir }}$.

in SPH. This lower cooling at the virial radius in SPH then leads to an inflated post-shock region. This is similar to the comparison of the projected temperature of a galaxy halo taken from the SPH code GADGET with the moving mesh code AREPO reported in Nelson et al. (2013), in which the $\mathrm{SPH}$ virial shock was located at larger radii than in the moving-mesh case.

Phase diagrams of the gas at $z=5$ out to the virial radii are given in Figure 7 . These plots show many new features not seen in the NC results shown in Figure 4. The medium is now found mostly at 10,000-20,000 K, where the cooling function has a local minimum. As gas slowly cools through this regime, it becomes denser and therefore has lower entropy. At densities above $3 \times 10^{-24} \mathrm{~g} \mathrm{~cm}^{-3}$ the gas cools more efficiently, until cooling to the enforced polytope, where the temperature is forced to scale with $\rho^{1 / 3}$. Finally, post-shock gas is heated to just above the virial temperature and cools inefficiently except at high densities. The SPH-NC gas at high temperatures that extends to a density of $\rho \simeq 10^{-24} \mathrm{~g} \mathrm{~cm}^{-3}$ is able to cool in the SPH-FID simulation, forming lines extending down to the cooler, $T \simeq 10^{4} \mathrm{~K}$ regime. Finally, given that gas was found at slightly higher densities in SPH-NC than in AMR-NC, we naively expected the cooling rate for the SPH-FID gas to be faster. However, since the polytrope gas extends to higher densities and temperatures in AMR, SPH appears to prevent gas in the polytrope from moving to higher densities. This may be due to undermixing, as discussed before. Additionally, AMR creates star particles of smaller mass than in SPH, thus AMR is more quickly removing pressure support from the densest regions, possibly leading to the buildup of more highdensity polytrope gas. A future study of star particle mass and SFR is needed to better understand this effect.

Next we carry out the same analysis at $z=3$, which we will also use to compare with the QSO runs, in the redshift regime where AGN feedback becomes more important. Density and temperature projections of the gas at this redshift are given in Figure 8. In density, the various accretion filaments have coalesced into a more condensed cosmic web that is less volume filling, with denser gas in the cosmic nodes. The temperature projections also show an increased virial temperature that extends to a larger radius, and the filaments are also encased within post-shock heated gas. The virial shock radius in SPH and AMR are both now at or beyond the virial radius at this redshift since both the cooling time and the dynamical time 

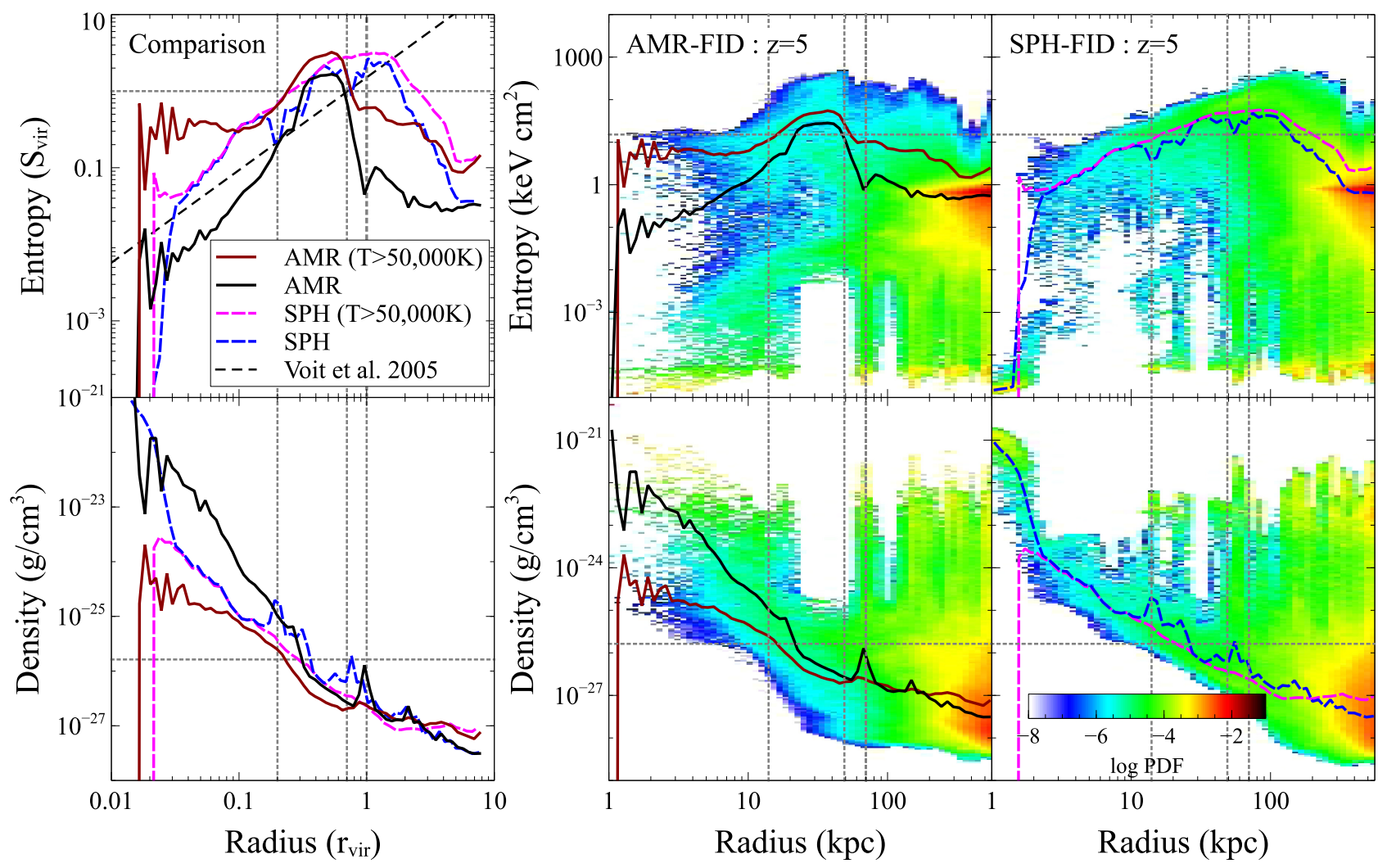

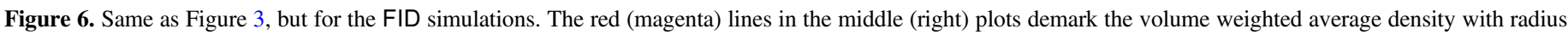

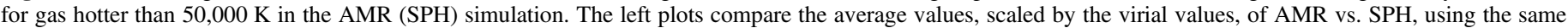
color and line scheme as the middle and right plots.

are roughly $0.11 \mathrm{H}^{-1}$. In AMR the filaments clearly remain relatively cold, while this is much more difficult to see in SPH. Looking at the temperature slices (right), indeed the filaments inside the shock-heated gas are cool in SPH, but they are composed of smaller, more fragmented, gas clumps than in AMR, while the post-shock gas is much more extended. The discrepant behavior, that is the overheating and undercooling at the shock, that explained the hotter gas in SPH at $z=5$ at the virial radius has had a runaway effect, and by $z=3$ its impact is even more extreme. In the galaxy-scale inset, it is clear that there is cold gas in both AMR and SPH, within which stars form, but this gas is more ordered in AMR, consistent with a higher sAM.

In the profile plots, presented in Figure 9, we see the same qualitative behavior in entropy and density for AMR and $\mathrm{SPH}$ as at $z=5$. A similar trend in the entropy of the hot gas is visible in the volume-average profiles: in particular, at small radii $\left(r<0.05 r_{\text {vir }}\right)$ the SPH entropy is lower than in AMR, at intermediate radii $\left(0.05 r_{\mathrm{vir}}<r<r_{\mathrm{vir}}\right)$ the SPH entropy is larger than in AMR, at $r \simeq r_{\mathrm{vir}}$ the entropies are in good agreement, and out to $2 r_{\text {vir }}$ the SPH entropy is again higher, with temperatures near the virial temperature. However, the ratio of AMR and SPH entropies are larger than their values at $z=5$, thus the behavior of the two codes is even less consistent. The densities, on the other hand, show the same level of consistency between the two codes at $z=3$ as at $z=5$.

Thus at both $z=5$ and $z=3$ with radiative cooling, star formation, and reionization, the overall trend is for standard SPH to have lower entropy cluster cores and larger entropy at larger radii compared with AMR. This is consistent with other comparisons between standard SPH and AMR for non- radiative simulations (e.g., Voit et al. 2005; Mitchell et al. 2009), but oddly, this is not what is seen in comparisons between standard SPH and the moving mesh code AREPO. In fact, Kereš et al. (2012) saw that for intermediate mass halos similar to our halo that the moving mesh simulations have even lower entropy values in the core and higher entropy values near the virial radius than the SPH runs. The authors argued that the moving mesh was capturing a cooling flow and thus SPH was not capturing sufficient cooling in the gas. While this may be the case, the authors also discuss the mixing of low entropy gas at the center of the cluster in AREPO which is not captured in GADGET. Yet Mitchell et al. (2009) compared SPH to Eulerian simulations to show that such mixing injects heat into the central region, leading to a higher, not lower, entropy value. While Mitchell et al. (2009) did not include cooling, we still find the same behavior in our AMR simulations with cooling, suggesting that dissipative heating is in fact sufficient to offset the cooling in the center. This is even with our assumed constant metallicity of one-third solar and an overestimate of the cooling from $\mathrm{H}$ and $\mathrm{He}$ (see Section 2).

The tendency for SPH to have more diffuse, high-entropy gas is made clearer in Figure 10. The extra heating occurring at the virial radius has led to higher entropy gas in the SPH run, and this material cannot cool on a Hubble time ( $S$ is above $100 \mathrm{keV} \mathrm{cm}^{2}$; Oh \& Benson 2003). In AMR, this gas instead only reaches $S=10 \mathrm{keV} \mathrm{cm}$. The temperature profiles again are in good agreement, although the post-virial shock gas does still extend to slightly hotter temperatures. Thus SPH locks up more gas in the diffuse, high-entropy phase, which will have an impact on the amount of star-forming gas in the cluster. Finally, in SPH there is a small feature extending from the star-forming 


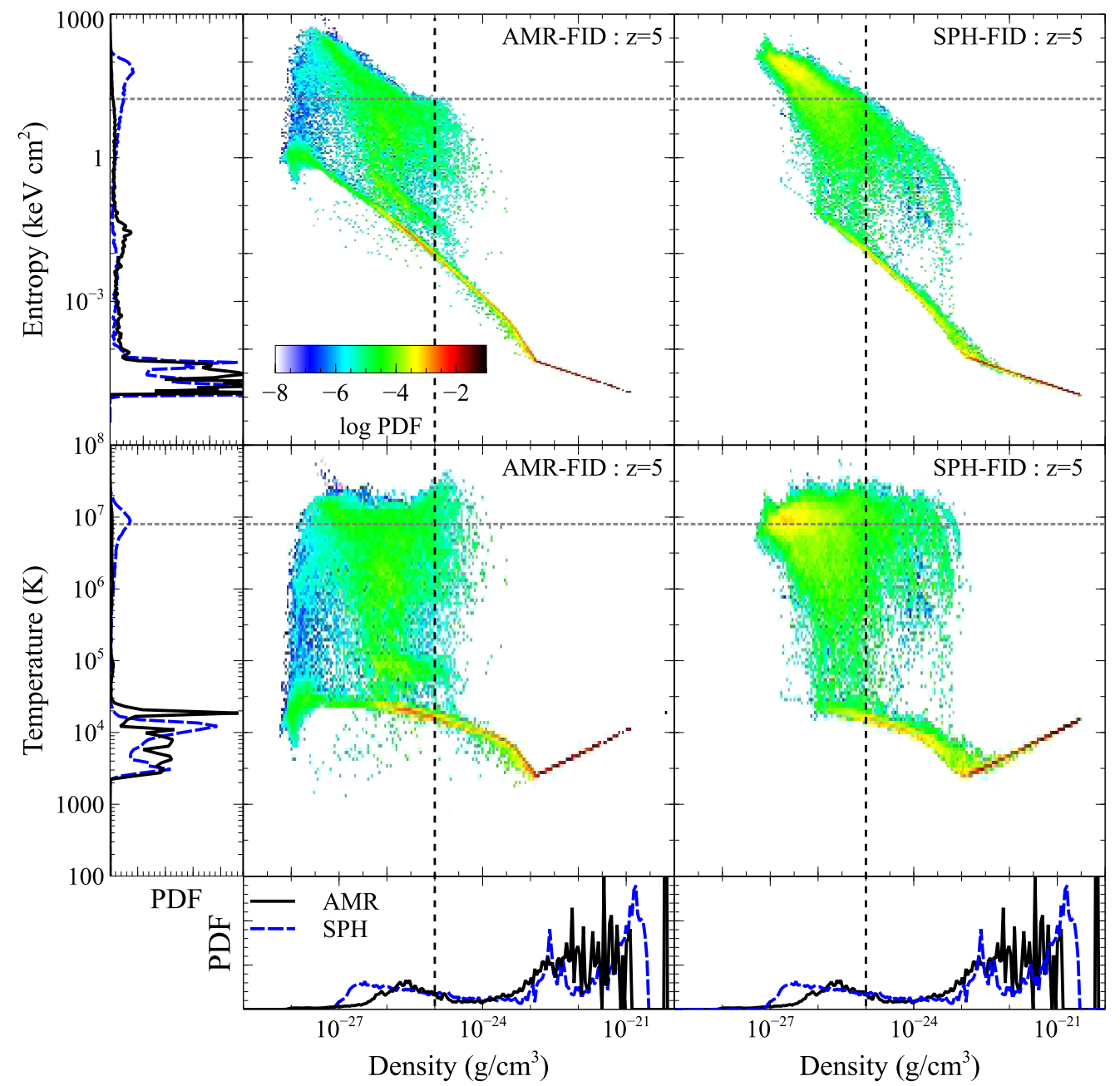

Figure 7. Same as in Figure 4, but for the FID simulations. The vertical dotted line marks the star formation density threshold at roughly $10^{-25} \mathrm{~g} \mathrm{~cm}^{-3}$.

ISM to hotter, denser gas. This is a post-SNe feedback region, where gas is instantly moved to higher temperatures in the phase diagram and then has its cooling artificially delayed. This results in the heated gas first expanding adiabatically, dropping in density and temperature, until as its cooling ramps up it cools more quickly and its entropy drops.

\subsection{AGN Feedback Runs}

We now look at the QSO simulations to see how the inclusion of our model of AGN feedback impacts the halo and galaxy gas and how AMR and SPH compare in this context. We first begin by comparing these results with the $z=5 \mathrm{NC}$ and FID results, followed by the FID results at $z=3$.

The halo virial quantities for these simulations are listed in Table 2. The inclusion of AGN feedback has led to a much lower baryon fraction than in the FID simulations, which is now consistent between the AMR and SPH simulations. As we show below, with AGN feedback, the virial shock is now beyond the virial radius in both AMR and SPH, and it is for this reason that the baryonic fraction has dropped, as gas spends a longer time in the outer halo before cooling and falling within the virial radius.

In the left column of Figure 11, we show large-scale (out to 8 $\left.r_{\text {vir }}\right)$ projections of the gas density for the AMR-QSO (top) and SPH-QSO (bottom) simulations at $z=5$, to compare with
Figures 2 and 5. The introduction of AGN feedback results in very little apparent impact on the density distribution at this redshift. This is mostly due to only a $30 \%$ drop in overall gas fraction, which is difficult to see with the scale spanning six orders of magnitude. However, under close comparison with Figure 5, we see that the densest regions in the QSO runs have the largest decrement in density compared with the FID runs, while the filaments inside the cluster are somewhat denser. In AMR we see that the filaments are slightly pushed off-center and are thinner. In SPH, there is a lower number of collapsed clumps, suggesting that the feedback is offsetting the numerical dissipation. Finally, the diffuse halo medium inside the virial radius is slightly denser with AGNs. Thus, while by $z=5$ AGN feedback is not effective in moving large amounts of matter out of the cluster environment, it does reduce the central densest peaks and delay gas accretion into the halo.

The middle and right columns of Figure 11 present projections and slices of the gas temperature, respectively. While AGN feedback does not affect the gas density, it clearly affects the gas temperature. In both simulation types, the AGN feedback results in a larger volume of hot gas than in the FID runs, with an increase in the hot gas volume-filling factor of roughly 10 , and a virial shock radius well beyond the virial radius. However, note that in both FID and QSO simulations the amount of heated gas is consistently greater for the $\mathrm{SPH}$ simulations. Given that more gas is heated in $\mathrm{SPH}$, it is 

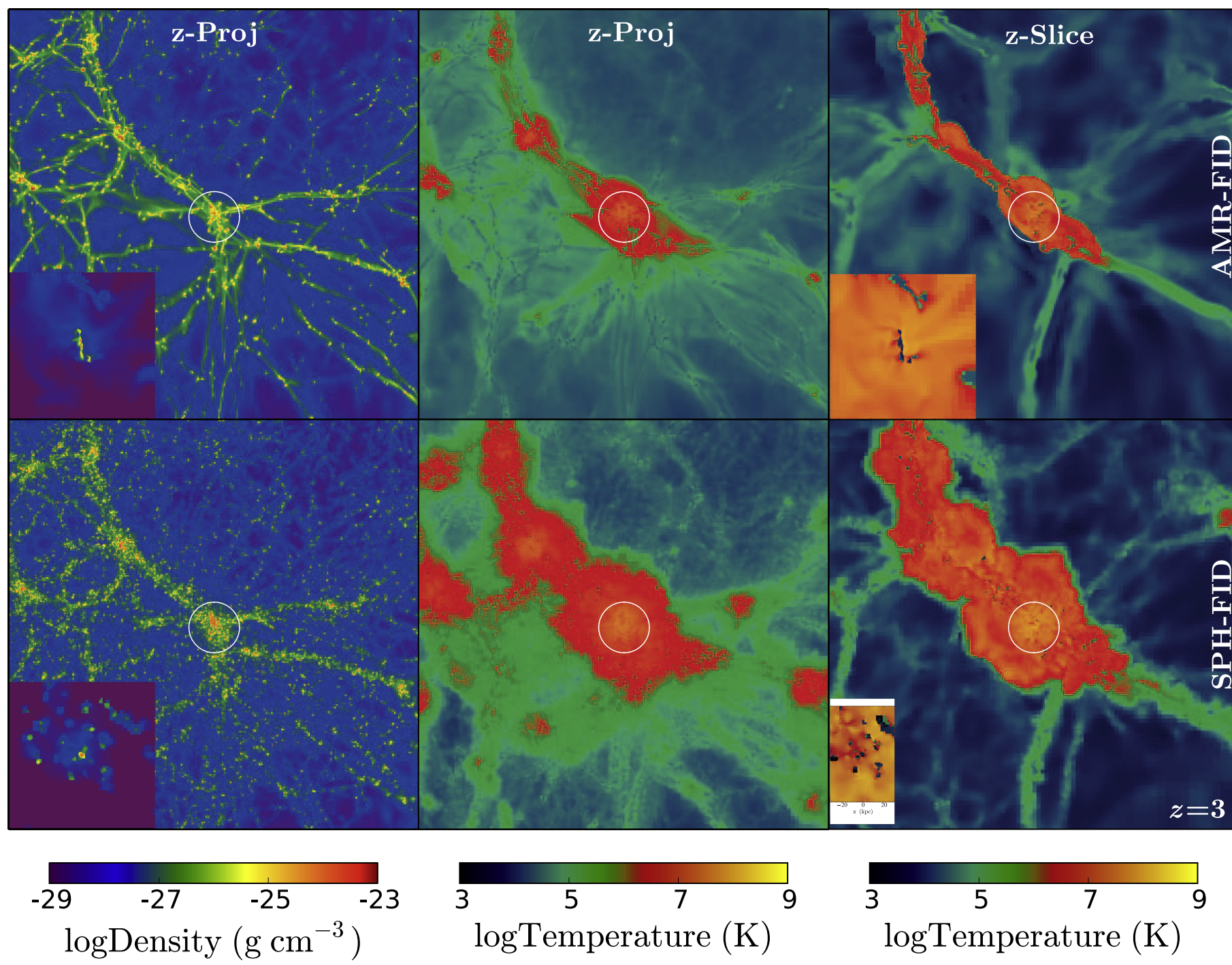

Figure 8. Same as Figure 5 but for $z=3$, where now $r_{\mathrm{vir}}=220$ physical kpc. The images thus measure about $3.5 \mathrm{Mpc}$ across centered on the halo.

thus surprising that the temperature slices reveal that the two methods give a consistent picture of the halo gas (within the white circle). The halo virial radii are 66 physical kpc and 70 physical kpc for AMR-QSO and SPH-QSO, respectively, and within the halo the gas is nearly uniform at the virial temperature of $8 \times 10^{6} \mathrm{~K}$. Although the AMR filaments are more continuous than in SPH, similar to what is seen in the FID simulations, the cold filaments in AMR-QSO truncate at a larger radius than in AMR-FID and point slightly away from the center of the cluster. The filaments' truncation radius is signified by a second shock located at the virial radius. Thus, although it is not visible in the large-scale projections, the AGN is impacting filament gas on small scales in the AMR simulation, which is also seen in higher resolution runs in Dubois et al. (2013). We do not see a similar impact on the filaments in SPH, however, and this is consistent with what is seen in Di Matteo et al. (2012).

We interpret the similarity of the AMR and SPH halo gas as follows: although the injection scale of the AGN feedback for the two methods is not identical, the AGN eventually heats the halo gas sufficiently to self-regulate its accretion, and this selfregulating gas configuration occurs at a "critical entropy" (Oh \& Benson 2003; Scannapieco \& Oh 2004). We find that this critical entropy is largely code independent. However, it is clear that SPH causes more collateral gas heating, such that the gas at very large radii is heated to $10^{5} \mathrm{~K}$ in the process of increasing the halo gas to the same critical entropy. This becomes much clearer at $z=3$, as discussed below.

Beyond the halo, we see that the intergalactic medium in the SPH simulation is significantly cooler than in the AMR simulation. This difference is unfortunately caused by an erroneous switch that turned off reionization heating for gas below $500 \mathrm{~K}$ in our SPH-QSO simulation. However, the shock increases the temperature in AMR by three orders of magnitude, and therefore the fact that the IGM gas is artificially cooled results in little impact on the halo itself.

The impact of AGN feedback on the gas profiles of the cluster environment at $z=5$ is presented in Figure 12. Even though the temperature projections show a strong signature of AGN feedback in both simulations, the average profiles in this figure are largely similar to the fiducial cases shown in Figure 6. However, in the QSO simulations, the distribution of gas at very high entropies has increased, extending roughly an order of magnitude higher than in Figure 6, with the largest increases occurring at very small radii $(r<20$ physical kpc) and very large radii ( $r>100$ physical kpc). Additionally, the 

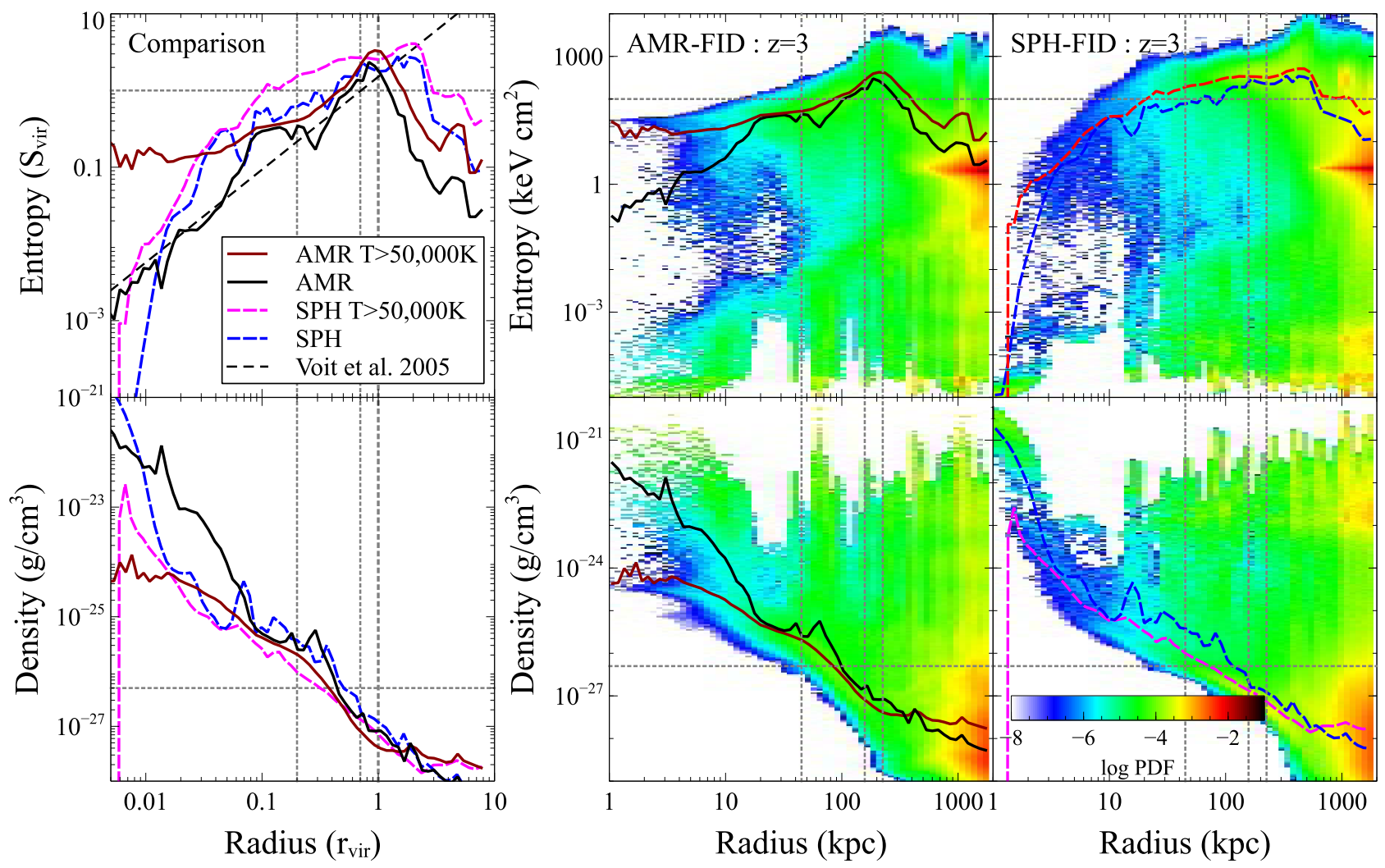

Figure 9. Same as Figure 6, but for $z=3$.

inclusion of AGN has resulted in less low-entropy gas at $10-20 \mathrm{kpc}$ in the AMR simulation, while in the SPH simulation there is less low-entropy gas $\left(S<0.001 \mathrm{keV} \mathrm{cm}^{2}\right)$ at all radii, but more mid-entropy gas $\left(S \simeq 0.1 \mathrm{keV} \mathrm{cm}^{2}\right)$ at 5-20 physical kpc. Finally, by comparing the QSO results with the nonradiative profile given in Voit et al. (2005), we see that the inclusion of AGN feedback has resulted in a higher average entropy value at all radii for AMR, and a higher average entropy for the central gas in the SPH case.

The AGN feedback modeled here acts to diminish the SPH and AMR central density values and average values, bringing the two methods into better agreement. However, the hot SPH gas extends to higher densities, indicative of a very recent feedback event in which the gas has not yet had an opportunity to expand. This central, hot, feature is visible in the insets shown in Figure 11, which also show the presence of more cool gas in SPH, explaining the lower entropy profile. Overall there is much better agreement between the two methods than in the FID runs, where the AGN heating results in similarly increased entropy profiles necessary for selfregulating their accretion.

In Figure 13 we show the phase diagrams of the $z=5$ gas within the virial radius. The one-dimensional PDF plots of the entropy make it much clearer that the amount of high-entropy gas has increased by including AGN feedback. In AMR-QSO, much more gas has $S \geqslant 100 \mathrm{keV} \mathrm{cm}^{2}$ than in AMR-FID, and this high entropy gas is found over a range of densities, from $\rho=3 \times 10^{-27} \mathrm{~g} \mathrm{~cm}^{-3}$ to the star formation threshold density of $10^{-25} \mathrm{~g} \mathrm{~cm}^{-3}$. In SPH, there is a smaller increase in gas with $S \geqslant 100 \mathrm{keV} \mathrm{cm}^{2}$, and most of this is at low densities $\approx 3 \times 10^{-27} \mathrm{~g} \mathrm{~cm}^{-3}$. Thus, within the halo, AGNs are heating gas at a range of densities in AMR, preventing it from cooling to very high densities, while in SPH, AGNs are more efficient at heating the surrounding diffuse gas. This increase in highentropy, hot gas is also shown in the one-dimensional PDF plots of temperature, which illustrate that in the AMR case the AGNs heat less gas but this gas is heated to higher entropies and temperatures than in the SPH case. This is partially due to the way the feedback energy is injected into the simulation, with AMR distributing the energy to the neighboring cells of the $\mathrm{BH}$ particle, and SPH distributing the energy over the $\mathrm{BH}$ particle's smoothing length.

Finally, as shown in the profile plots, in SPH the AGN is causing significant heating of dense gas in the polytrope. This gas occupies the high-density region of the SPH plots, where the very dense but also hot gas is unphysical. Yet it appears to be a long-lived feature. This occurs when a sink particle resides in a cold, compact, dense clump (such as seen in the inset of Figure 5), but it is not sufficiently massive for Eddingtonlimited feedback to overcome the clump's binding energy. Instead, the AGN must grow while keeping the clump at a hot temperature, until finally dumping sufficient energy into the clump to overcome gravity.

Moving to $z=3$, projections of gas density and projections and slices of gas temperature out to $8 r_{\mathrm{vir}}$ are shown in Figure 14. We see the same small effects as at $z=5$. Thus, even at later times when AGNs have a larger impact, thermal feedback does not lead to large-scale redistribution of gas. The temperature projections now present significantly more extended hot gas, which, if we consider all gas heated above the typical IGM temperature of $10^{4} \mathrm{~K}$, is more volume filling in $\mathrm{SPH}$. However, if we consider the region with projected temperature above $10^{6.5} \mathrm{~K}$, then we again see that there is fairly good agreement between the two methods. This region in 


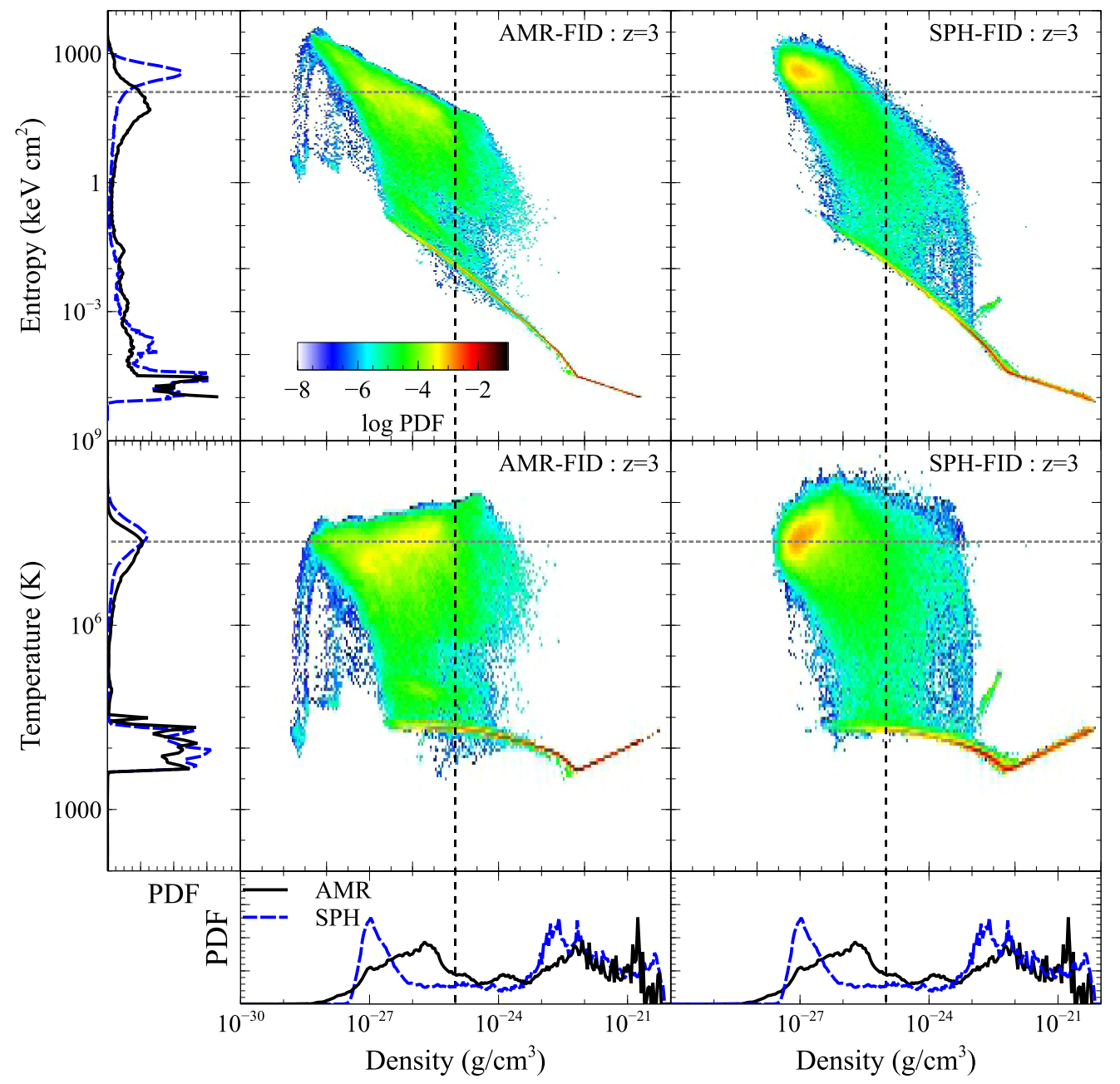

Figure 10. Same as in Figure 7, but for $z=3$.

SPH is only slightly larger and reaches temperatures only slightly hotter than in AMR. Thus we find that the methods produce a halo gas temperature distribution that is much more consistent than in the $z=3$ FID runs and the QSO runs at $z=5$. By $z=3$ the AGN has heated the gas to roughly the same entropy in both methods, the entropy required for selfregulation.

This agreement on intermediate and large scales aside, the inserts, showing the gas density and temperature in the inner $0.2 r_{\text {vir }}$, do show a few differences. Thus while the AGN feedback acts to make the bulk of the halo material consistent thermodynamically, on smaller scales the gas is impacted differently. In SPH there is a more centrally collapsed, hot, gas clump, which is broader in density than in temperature, and whose outskirts are denser than its interior. This region is in the process of a feedback-driven expansion, leading to a shock front with higher density and therefore increased cooling. This expansion phase is made clearer in the gas profiles shown in Figure 15. In SPH the gas density peaks outside of the center at roughly 4 physical $\mathrm{kpc}$, where entropy drops. We have confirmed that this is not due to an improper choice of halo center.

On intermediate and large scales in Figure 15 there is again better agreement between the two methods. Outside of the central clump, the AGN has led to a much larger amount of gas at high entropy at large radii, compared with the FID simulations in Figure 9. Within the virial radius, the average entropy and density profiles are even more consistent than in the FID and $z=5$ QSO runs. Given that in SPH there is a central clump with higher density, we would expect to see lower entropy in the core of the SPH halo. However, the gas has finally been heated sufficiently to drive an outflow, giving a central peak in the SPH entropy. We have looked at $z=3.1$, and found at this earlier time that the central SPH entropy is indeed lower than in AMR. Thus the inversion at $z=3$ is a recent event, and in general lower entropy cores in SPH are more common.

Finally, in Figure 16 we compare the phase plots at $z=3$ for the cluster. We can see the expanding central clump in the SPH diagram, where a large plume of isopycnic gas has continuously been heated via AGN feedback until reaching a sufficiently high temperature that the pressure can unbind the clump. However, note that although this unphysical region appears quite large, it contains only a small fraction of the gas in the halo. Instead, a greater amount of gas is found near $10^{-27} \mathrm{~g} \mathrm{~cm}^{-3}$ in both methods. The AGN feedback has led to similar amounts of gas in the hot diffuse halo, although in AMR there is more gas held at $10^{4} \mathrm{~K}$ just below the star formation density threshold, while this gas is found above this threshold in SPH. This is due to the clumping nature of the cold gas in SPH, where hot gas is less able to disrupt these clumps through instabilities. In AMR, on the other hand, the dense gas 


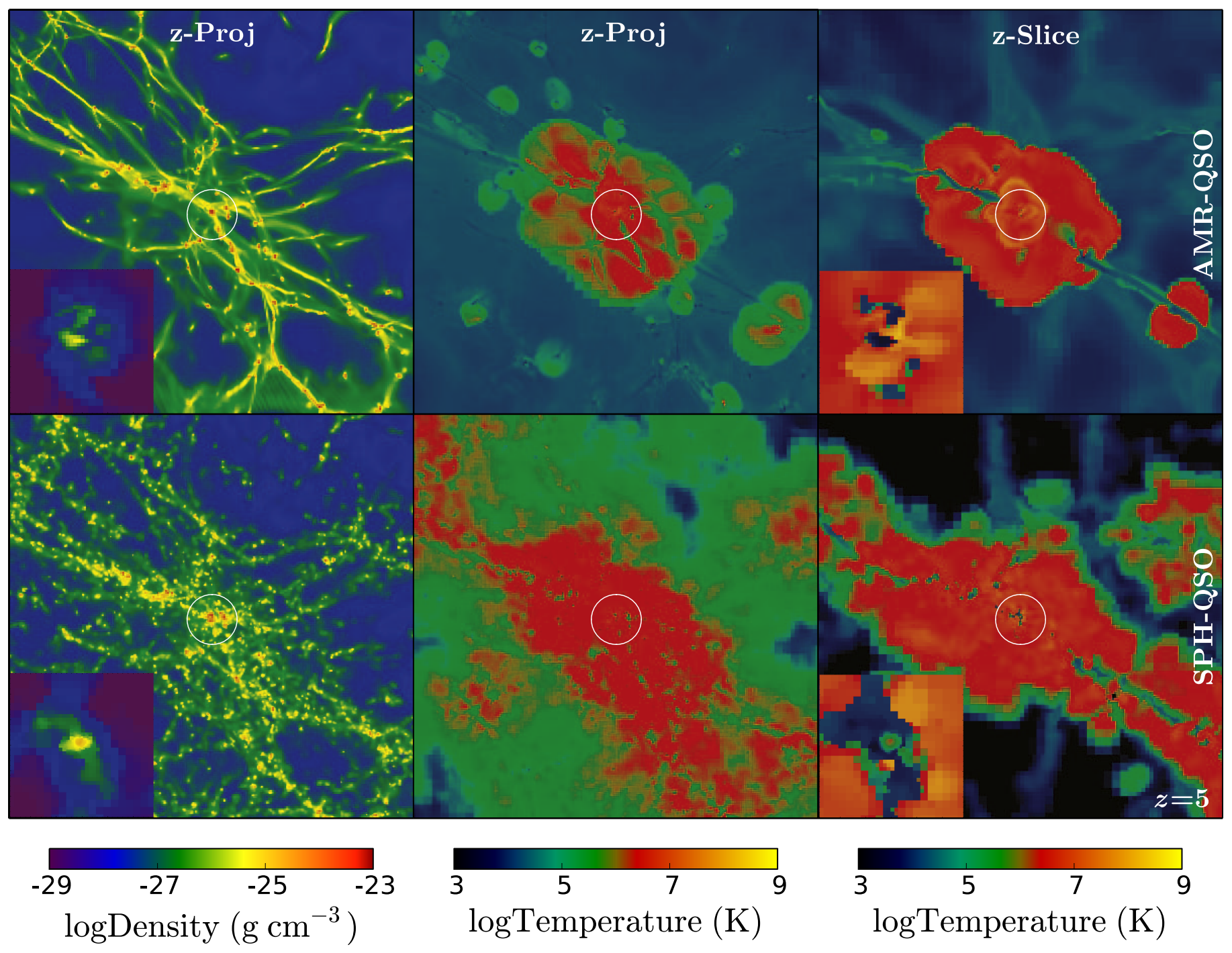

Figure 11. Same as Figure 5 but for the QSO simulations.

forms streams instead of clumps, with larger surface area that is more susceptible to turbulence. Thus in AMR the AGN feedback is better able to dissolve and/or physically move the very inner filamentary gas into the warmer, diffuse medium, consistent with what has been seen in previous work (e.g., Dubois et al. 2013; Nelson et al. 2015).

To conclude this section, in Figure 17 we show radial plots of the cumulative baryon and gas mass fractions of the FID and QSO halos at $z=3$. The inclusion of AGN feedback has resulted in a lower cumulative baryon fraction at almost all radii, with the decrement largest near $r_{7000}$, where the average density within $r_{\mathrm{N}}$ is $N$ times the critical density. We include $r_{7000}$ here because $r_{2500}$, which is more traditionally used for comparing the inner halo, lies at the same radius as a large subhalo. We see that at $r_{200} \mathrm{AGN}$ feedback leads to a decrement in the baryon fraction of about $27 \%$ for both AMR and SPH, which increases to $34 \%$ and $31 \%$ at $r_{500}$, respectively, and $39 \%$ at $r_{7000}$. Figure 17 also shows that SPH-FID has less baryons than in AMR-FID, consistent with our previous discussion of these baryons spending a longer time beyond the virial radius. Also, the outflow in SPH-QSO is clearly seen in the bump in baryon and gas fraction at $4 \mathrm{kpc}$. In contrast to Sembolini et al. (2016a), the gas fractions for both of our AGN runs have decreased, at all radii beyond the SPH-QSO outflow. This is consistent with some combination of two effects. The first of these is that our feedback prescription is perhaps more effective at preventing the accretion of gas, while the second effect could be that our FID stellar mass fractions are already considerably lower than the simulations in Sembolini et al. (2016a), leaving little gas to be prevented from forming stars. A more thorough discussion of our stellar content is presented below, and in (M. L. A. Richardson et al. 2016, in preparation). We urge future studies to include both the baryonic and gas fractions of their halos, to better interpret how AGN feedback affects the gas component.

In summary, the two simulation methods produce a halo environment that shows the best agreement when we include AGN feedback. This is consistent with our AGN feedback model resulting in a self-regulation scenario, which becomes stronger at later times. The AGN heats the halo environment to sufficiently high entropies so that it turns off further gas accretion. It is very interesting that, even though the two methods cannot probe precisely the same spatial scales, the resulting entropy for which self-regulation occurs is very similar. Finally, on large scales in SPH, however, the result of AGN feedback heating the halo gas to this self-regulatory 

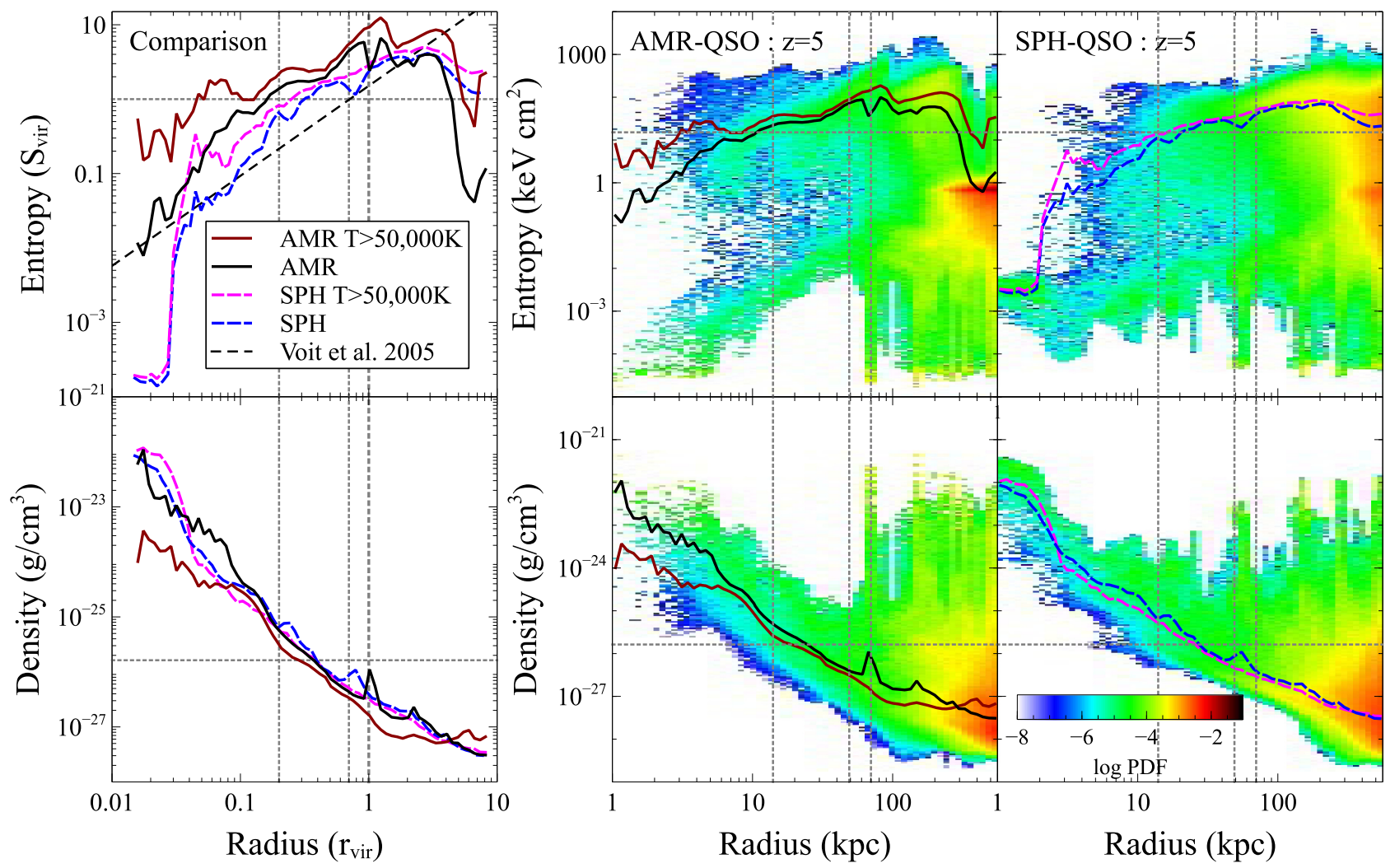

Figure 12. Same as Figure 6 but for the QSO simulations.

temperature is that a much larger volume of gas is also impacted. This would have interesting implications for surveys of the thermal Sunyaev-Zel'dovich effect (e.g., Spacek et al. 2016).

\subsection{Evolution of Gas, Stars, and Black Holes}

\subsubsection{Halo Gas}

Finally, we turn to a more detailed study of the halo's evolution. In Figure 18 we summarize the mass history of the halo, taken from a range of outputs from roughly $z \simeq 5-3$. The total halo mass evolution is consistent with pure exponential growth, similar to that seen for a range of halos in Wechsler et al. (2002). Using an offset at $z=3$, so that the leading constant is roughly the mass of the halo at the end of our simulations, we get an equation of the form

$$
M_{\mathrm{tot}}(z)=M(z=3) e^{-\alpha(z-3)}
$$

where we find $M(z=3)=2.3 \times 10^{13} M_{\odot}$, and $\alpha=1.15$. This $\alpha$ value is consistent with the most massive objects considered in Wechsler et al. (2002). By breaking up the mass into components, we see the dark matter mass is more consistent between the AMR and SPH runs than the baryons. The AMR-FID run has a higher baryon fraction than the cosmic mean at all times, but this fraction drops with time. This is due to the virial temperature increasing as the cluster grows, leading to longer cooling times at further radii, slowly depleting the amount of baryons that fall inside the halo. In the SPH-FID run we see similar behavior, with the gas fraction dropping in time. However, the gas fraction starts out at roughly the cosmic mean, and then drops more quickly than in AMR. This difference is consistent with a higher post-shock temperature in $\mathrm{SPH}$ that is at a larger radius than in AMR. The inclusion of AGN feedback has little impact on the parameterization of the halo's growth. By looking at the effective slope, we can see there is a bit more spread in the QSO runs, with a slightly shallower $\alpha$ at large redshifts that becomes steeper at lower redshifts.

In the evolution of the halo mass, only the baryons appear affected by AGN feedback, resulting in a drop of roughly $30 \%$ in the amount of baryons in the cluster. With AGN feedback, there is more scatter in the evolution of the baryon fraction, but the AMR and SPH results are more consistent than in the FID runs. As we have seen, the AGN leads to roughly the same halo gas entropy, thus better agreement in the baryon gas fraction is expected.

The sAM, $j$, and spin parameter, $\lambda=j /\left(\sqrt{2} r_{\text {vir }} v_{\text {vir }}\right)$ (e.g., Bullock et al. 2001), are presented in Figure 19, where $v_{\text {vir }}$ is the virial velocity. The sAM of dark matter in the outer halo $\left(r>0.1 r_{\text {vir }}\right)$ grows at a smooth rate over the course of the simulation. Except at very early times, the growth of sAM is unaffected by AGN feedback. By normalizing by the virial radius and velocity it is clear that $\lambda$ for the outer halo is roughly constant in time with a value near 0.034 , consistent with tidal torque theory (e.g., White 1984; Bett et al. 2007). Accreting dark matter slowly exchanges its SAM with the halo, leading to a slower rate of growth of sAM in the central $0.1 r_{\text {vir }}$, corresponding to the galactic scale. Even on small scales the growth of sAM is not impacted by AGN feedback. As seen in Danovich et al. (2015), the dark matter sAM on small scales is roughly an order of magnitude below the accreting dark matter value. 


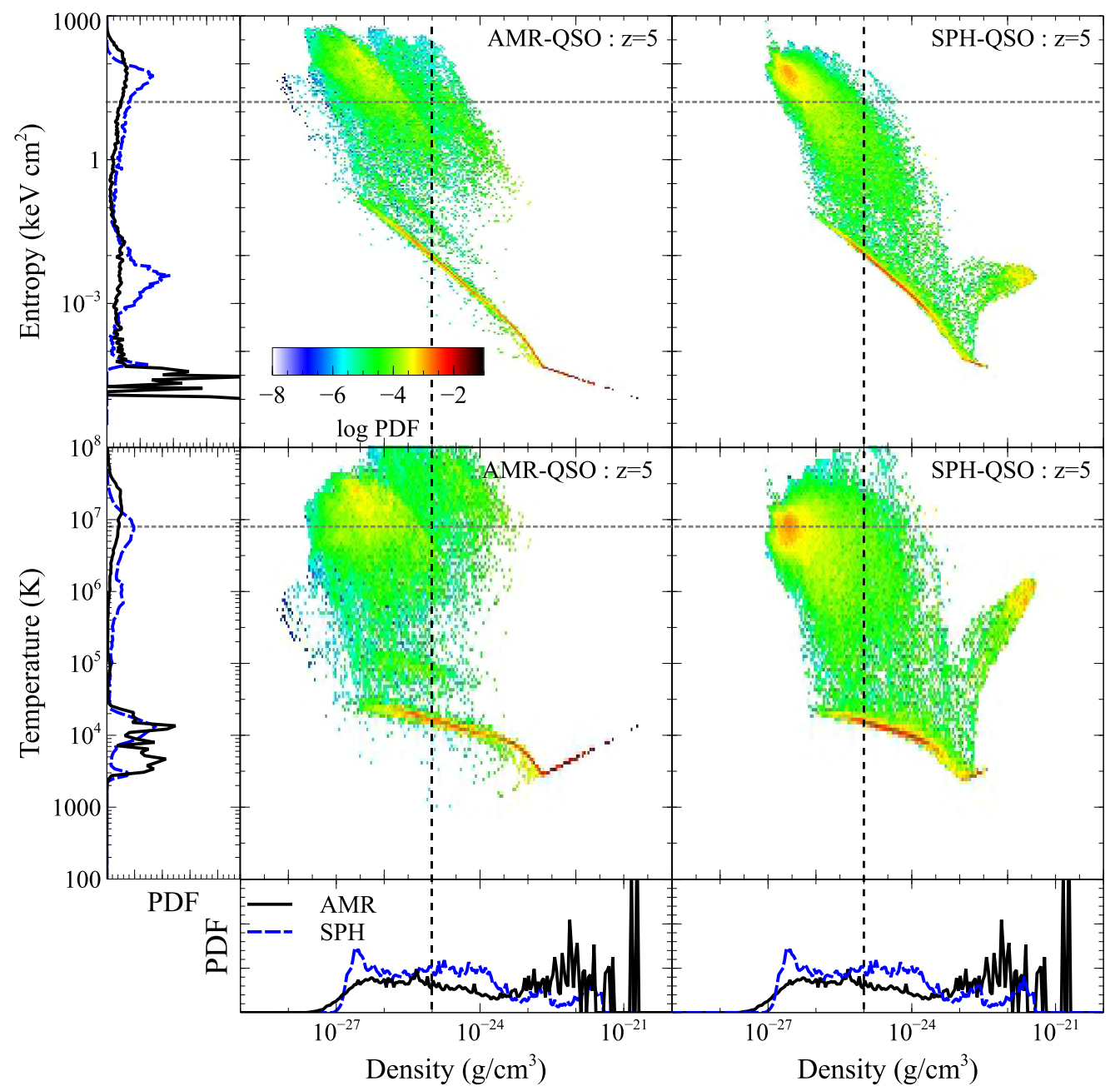

Figure 13. Same as in Figure 7, but for the QSO simulations.

The gas sAM behaves quite differently. Before $z=4$ the sAM is roughly constant in the outer halo, until at $z=4$ it has roughly the same sAM as dark matter, at which point it grows faster than the dark matter sAM. By $z=3$ the gas sAM is a factor of three above that of dark matter. At very early times, the lower gas fraction in the QSO simulations results in lower sAM. This suggests that at early times much of the angular momentum generation is via accretion of high sAM gas. However, by $z=4$ the outer gas sAM has the same value in all simulations, which suggests that a significant amount of the torque generating the angular momentum is gravitational in nature, consistent with tidal torque theory. The spin parameter is also roughly constant for gas in the outer halo, staying a factor of roughly two above the dark matter, except at $z=4$. Considering we are presenting the angular momentum of all gas, and not just cold gas, it is encouraging to see the agreement with Danovich et al. (2015), who see that cold gas in the outer halo has spin parameters of roughly three times that of dark matter. A more detailed study of the angular momentum of the different gas phases is beyond the scope of this work.

At small radii, however, there are significant differences between SPH-FID and AMR-FID, where the SPH sAM is consistently lower than in AMR. While SPH explicitly conserves angular momentum in the absence of artificial viscosity, Okamoto et al. (2003) demonstrated that due to its difficulty in modeling the layer between different fluids, standard SPH can transfer angular momentum from the dense disk to the hot diffuse halo. Additionally, Kaufmann et al. (2007) showed that disks in low-resolution SPH simulations, of comparable resolution to this and other cosmological simulations, unphysically transport angular momentum to the outer medium. However, AMR does not explicitly conserve angular momentum, its grid has a preferred direction, and it suffers from advection errors. Thus AMR also suffers from spurious angular momentum dissipation. Yet, we see here that the central $0.1 r_{\text {vir }}$ has an order of magnitude more angular momentum in AMR than in SPH. Thus, the SPH resolution issues may be playing the dominant role. Additionally, it is possible that the more collimated filament streams in AMR incur more sAM from tidal torques since it has a high quadrupole moment (e.g., Danovich et al. 2015), which is also weakly supported at high $z$ and large radii.

The inclusion of AGN feedback also leads to markedly different behavior in the inner region for the two methods. In $\mathrm{SPH}$, the inclusion of feedback has led to a combination of accretion of low angular momentum gas, and removal of this low AM gas from the central region. This leads to a net increase in the central SPH sAM. However, in AMR the sAM drops when we include AGN feedback. The two mechanisms leading to an increased sAM in SPH are also operating in AMR. Thus the difference is that gas heated via AGN feedback moves the cold stream filaments outward, inhibiting gas 

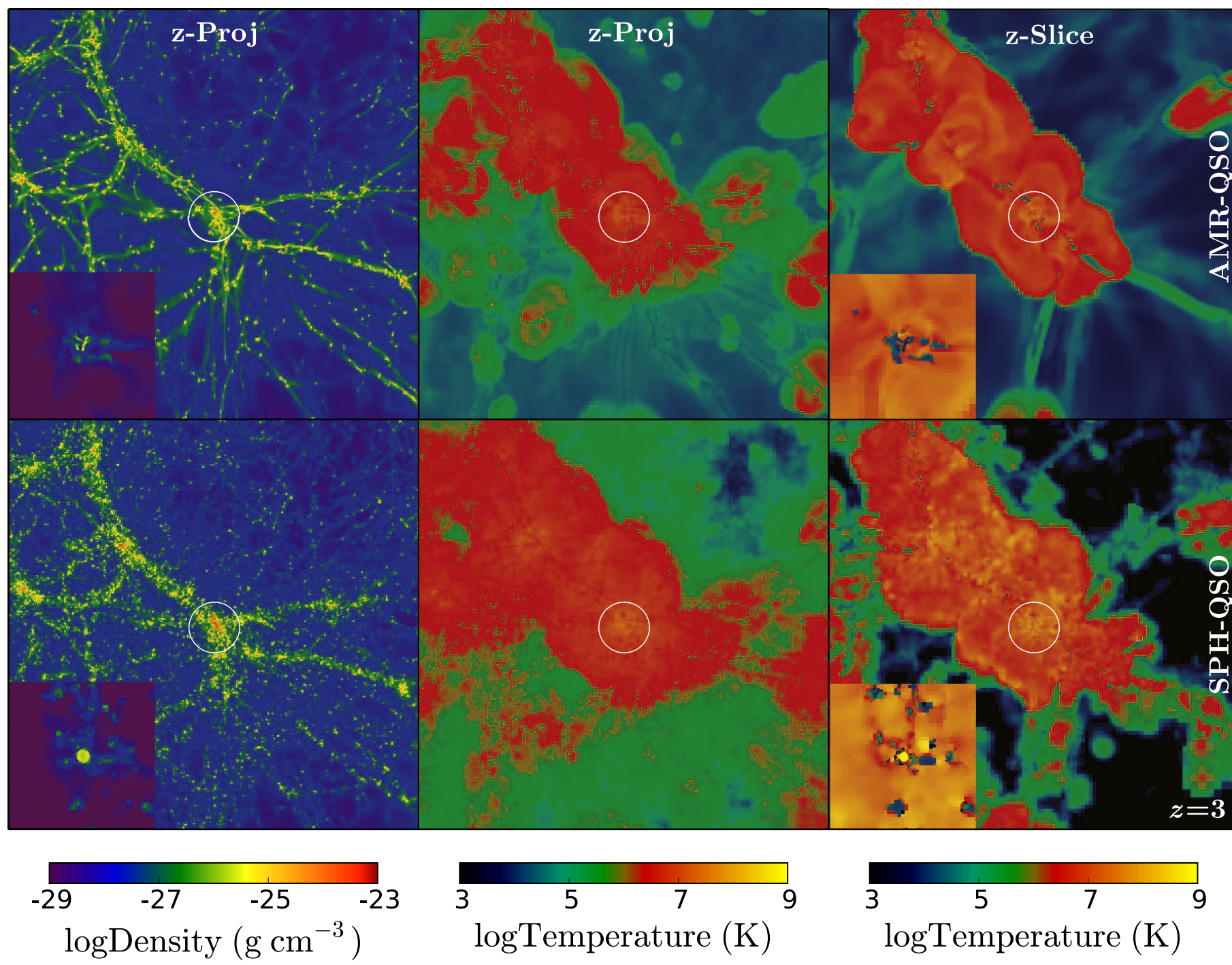

Figure 14. Same as Figure 11 but for $z=3$.

accretion from these outer regions and transfering the filamentary gas' angular momentum through shocks into the outer halo. While the sAM is increasing for all simulations, the spin parameters are dropping. Thus the angular momentum buildup in the central region is not keeping pace with the growth of the cluster, regardless of feedback processes.

\subsubsection{Star Formation}

In Figure 20 we present the halo stellar mass and SFRs for the four simulations containing stars. We fit the halo stellar mass functions with exponential power laws, similar to the total halo mass fits in Section 3.4.1. The values for the fit are given in Table 3. Up to $z=3$, it is always the case that the AMR simulations have more stars than in the SPH simulations. This is mostly due the gas reaching higher densities in AMR, as shown in Figure 7. Since the SFR scales with $\rho^{1.5}$, the higher density gas in AMR leads to a much larger stellar masses. The inclusion of AGN feedback leads to a significantly reduced amount of stars, due to removal of low angular momentum gas, and heating of surrounding halo gas. Unsurprisingly, AGNs have the biggest impact in SPH where there is an average increase in the central angular momentum and more large-scale heating, which reduces the SFR in surrounding structures outside the virial radius, and thus a lower amount of stellar mass is accreted.

The average SFR is provided in Figure 20 as well, taken as the change in halo stellar mass divided by the change in time between snapshots. We include the parametric fits, equivalent to the derivative of the exponential fits, given by

$$
\begin{aligned}
\frac{d M_{*}}{d t}= & \alpha_{*} M_{*} H_{0}(1+z) \sqrt{\Omega_{M}(1+z)^{3}+\Omega_{\Lambda}}, \\
= & 14.3 M_{\odot} \mathrm{yr}^{-1}\left(\frac{\alpha_{*}}{2}\right)\left(\frac{M_{*}}{10^{11} M_{\odot}}\right)\left(\frac{h}{0.7}\right) \\
& \times(1+z) \sqrt{\Omega_{M}(1+z)^{3}+\Omega_{\Lambda}},
\end{aligned}
$$

where $\alpha_{*}$ is the exponential slope parameter, and $M_{*}$ is the stellar mass at a particle redshift, which follows an exponential growth fit equivalent to Equation (4), whose parameters are given in Table 3. The SFR is consistently lower in SPH, but grows faster than in AMR. Since the rate is lower, there is more gas present at later times from which to form stars in SPH. For the FID runs, this is consistent with a longer cooling time in 

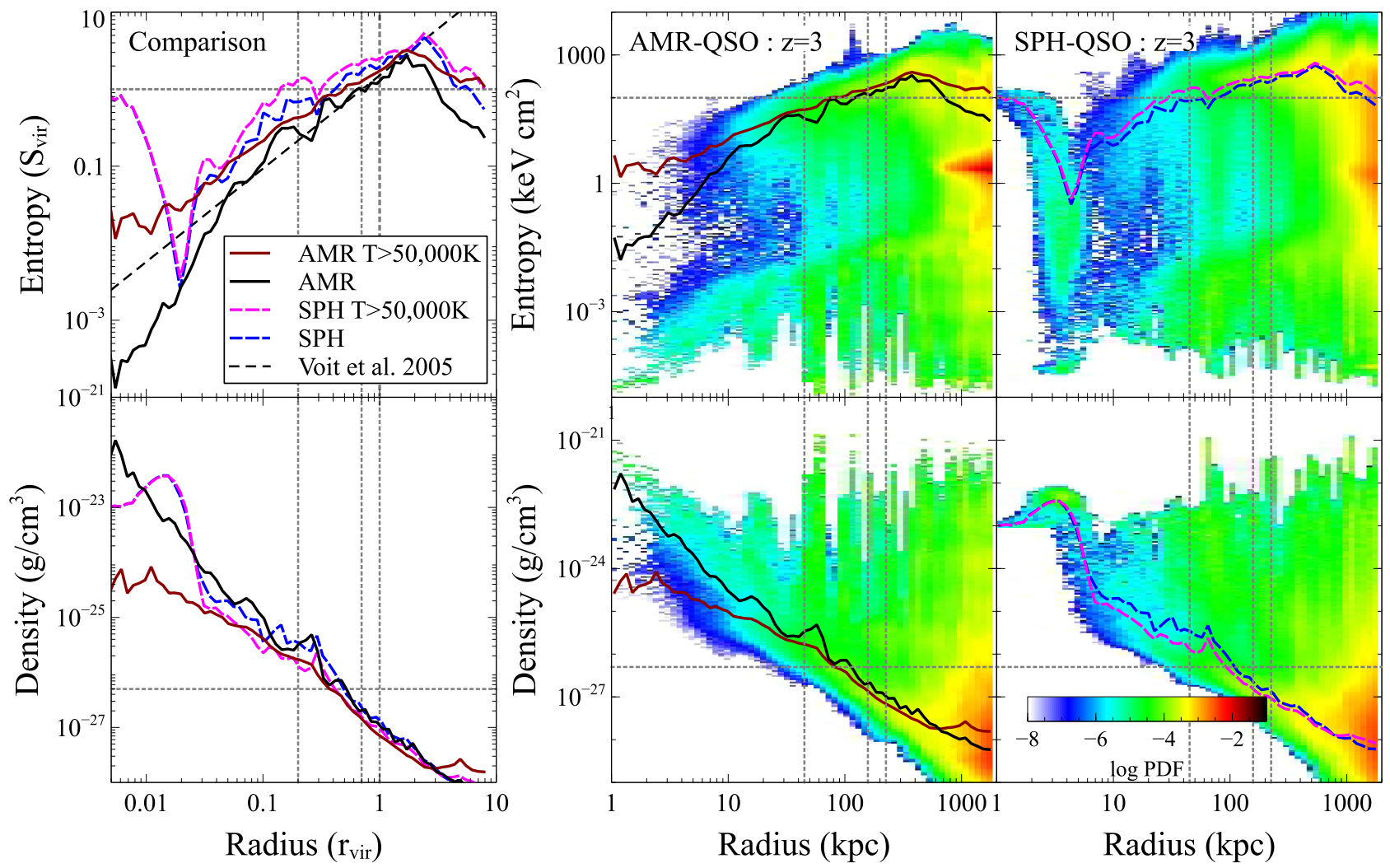

Figure 15. Same as Figure 12, but at $z=3$.

SPH. In the QSO runs, the SPH feedback has a larger impact on large scales than in AMR, thus the SFR is lower at high redshifts. However, at late times the halo gas becomes consistent between the two methods, which results in the SFRs approaching better agreement.

\subsubsection{Cluster BH Growth}

In Figure 21, we show the evolution of the sum of the mass of the halo's three most massive BHs, and their net growth rate, both in absolute terms and normalized by the sum of their Eddington accretion rate, each given in Equation (3). Since the SFR is lower in the SPH runs, it takes longer before the stellar density reaches the threshold to trigger the formation of a $\mathrm{BH}$. Thus, the SPH BHs form later and are less massive at very high redshifts. Since BHs are generated at the limit of density resolution, this could be a large source of discrepancy between the two codes. It is thus very interesting that the halo environment is so similar at late times. Due to the delayed formation, the SPH BHs' total growth rate is near Eddington, until the BH masses approach that of their AMR counterparts, at which point the growth rates are in good agreement, consistent within a factor of two in absolute terms, or roughly the same value relative to their Eddington rate. This is consistent with the AGNs entering the self-regulation phase. At $z<3.2$ a medium-sized SPH BH falls into a clump (see Figure 14), where its accretion increases very quickly. This accretion will remain high until the pressure is increased enough to turn off accretion, and this is what occurs at $z=3$ in Figure 15. During the period of best agreement between the two methods, $3.2<z<4$, we note that the baryon fractions are also in very good agreement (see Figure 18), but we see almost no correlation between the SFR during this time. Unfortunately, without sampling the star formation over smaller intervals, we are unable to determine whether fluctuations in star formation correlate with fluctuations in BH accretion, suggested in other works (e.g., Dubois et al. 2013).

\section{CONCLUSIONS}

To better understand the impact of AGN feedback on the formation and evolution of a large cluster and how this evolution can be biased by numerical effects, we have simulated the growth of a cluster from identical initial conditions in two different numerical methods. Using the AMR code RAMSES and the SPH code HYDRA we have attempted to match their radiative cooling, star formation, stellar feedback, and their BH formation, growth, and energetic feedback processes, with the caveat that the fundamental differences between the methods will make modeling even simple structure formation not necessarily identical. By comparing these simulations with successively more complex baryonic physics, we have observed the following key points.

1. Regardless of the treatment of baryonic processes, SPH consistently has a lower central entropy profile than AMR, with the sole exception being directly after an energetic feedback event. While this has been seen before for non-radiative simulations, comparisons with moving mesh codes have suggested that SPH codes may have higher central entropy profiles.

2. Gas that is heated by the virial shock can efficiently cool at high redshift in simulations, although this cooling may not be captured numerically if an artificial viscosity is 


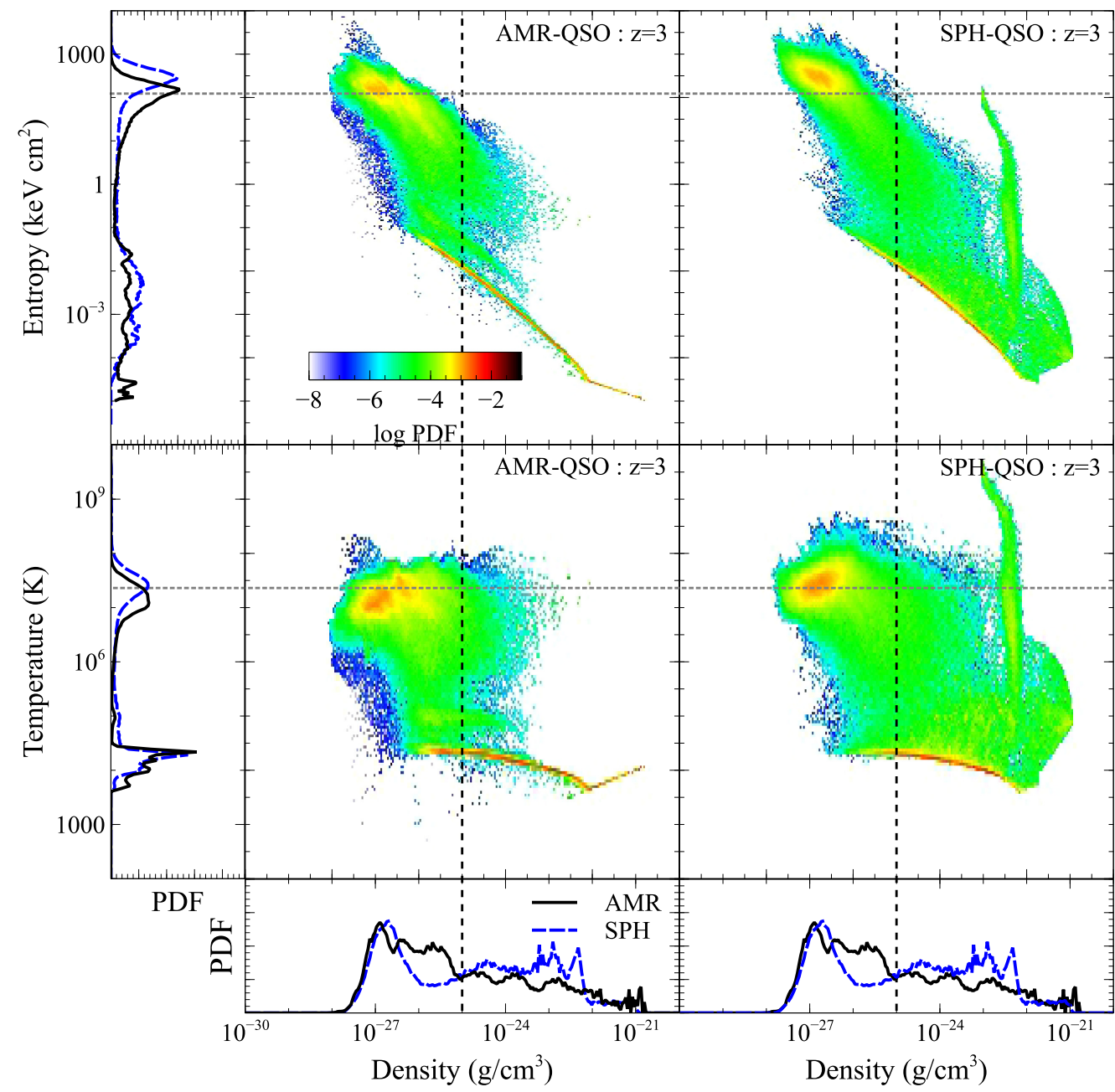

Figure 16. Same as Figure 13, but at $z=3$.

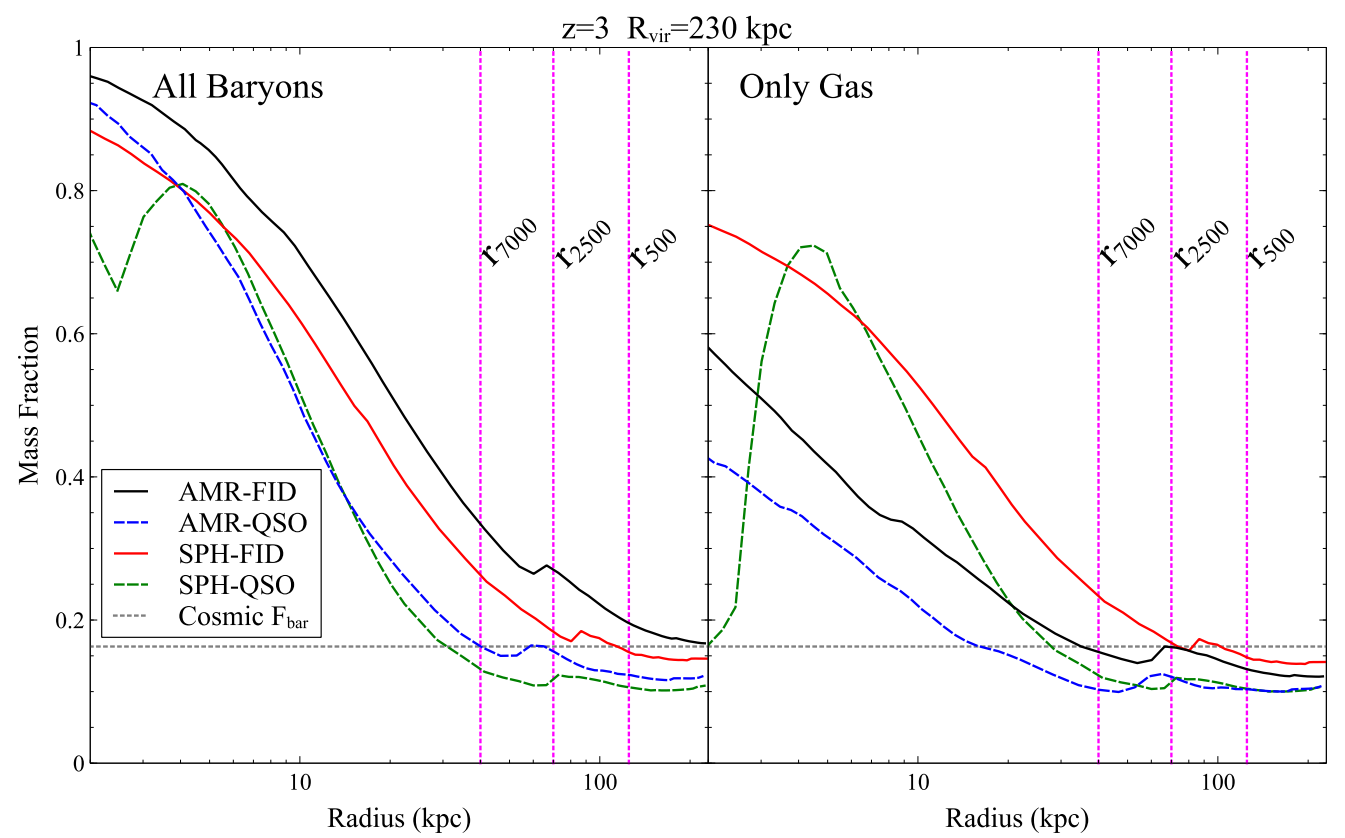

Figure 17. Radial profiles of the cumulative baryon (left) and gas (right) mass fractions of the FID and QSO halos at $z=3$ out to the virial radius $\left(r_{200}\right)$. The AMRFID (SPH-FID) simulation is presented with a solid black (blue) line. The AMR-QSO (SPH-QSO) simulation is presented with a dashed green (red) line. The horizontal gray dotted line is the cosmic average baryon fraction, and the vertical dotted magenta lines correspond to overdensities of 7000, 2500, and 500, from left to right, respectively. Recall that the virial radius as we define it in this work corresponds to an overdensity of 200, and thus the mass fraction values at an overdensity of 200 corresponds to the rightmost data points. 

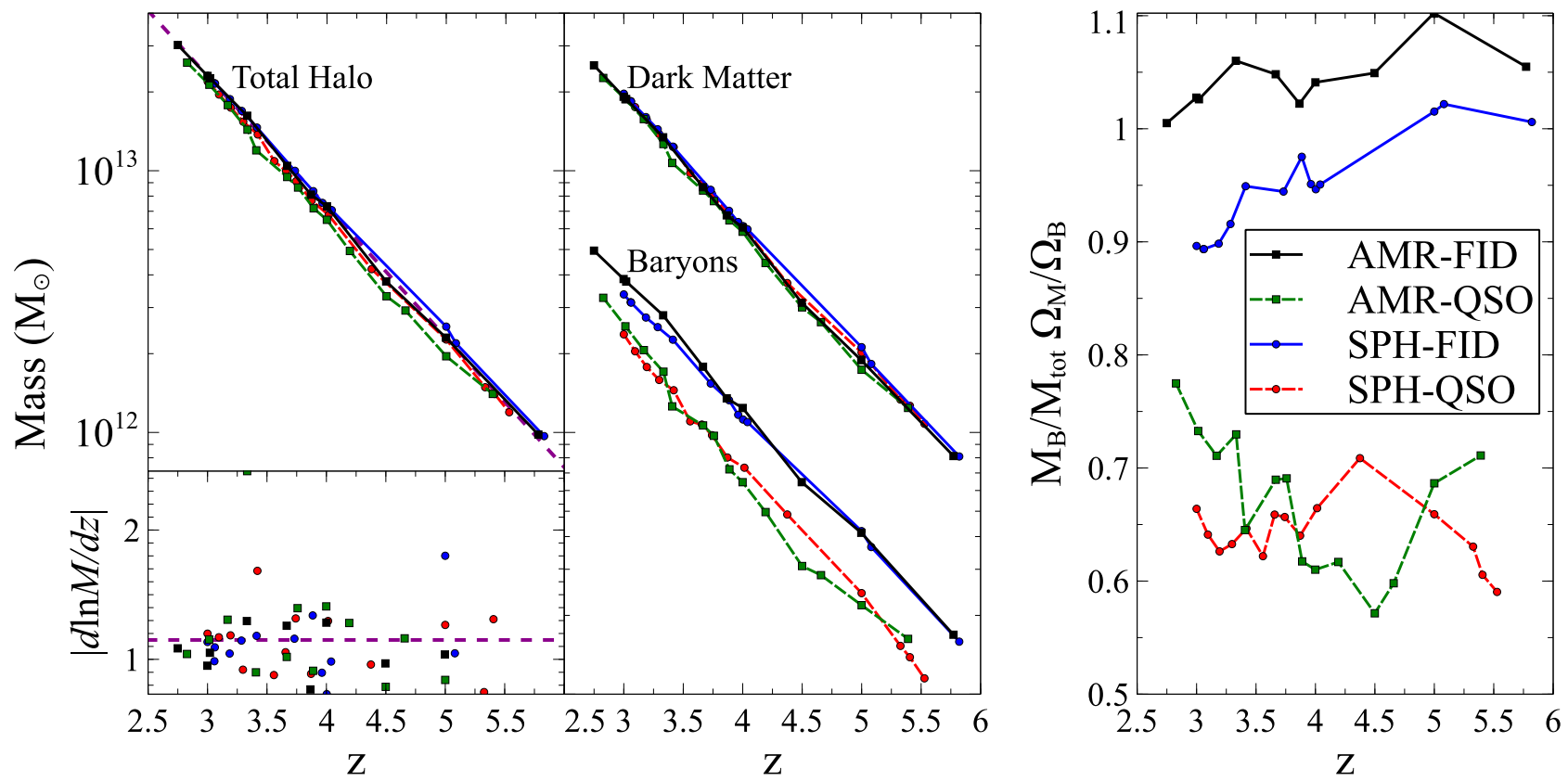

Figure 18. Top left: evolution of the total halo mass of the cluster. The AMR-FID (SPH-FID) simulation is presented with a solid black (blue) line where we mark the measured values in an output by squares (circles). The AMR-QSO (SPH-QSO) simulation is presented with a dashed green (red) line. The magenta dotted line gives an exponential mass law, $M=M_{\mathrm{f}} e^{-\alpha(z-z \mathrm{f})}=2.3 \times 10^{13} e^{-1.15(z-3)}$, following Wechsler et al. (2002). Bottom left: evolution of the halo mass accretion histories, given by $d \ln M / d z$. Line and point styles match the above plot, with the magenta line giving a constant value of 1.15, consistent with the exponential parameterization for $M(z)$. Middle: evolution of the dark matter and baryon components. Right: evolution of the gas fraction normalized by the cosmic mean baryon fraction.

employed or if the shock width is not well resolved. Future work studying the resolution dependence of this feature will shed further light on this issue.

3. The AGN feedback modeled here reduces the baryonic fraction of halos by roughly $30 \%$, regardless of the numerical method. The baryon fraction is highly dependent on the location of the virial shock, as it sets the bottleneck for subsequent baryonic accretion. The halo gas, being hotter and a small total mass, leads to a reduction in the total SFR of the cluster halo, by up to an order of magnitude by $z=3$. Future work comparing the two simulations methods at lower redshift is needed to determine if their apparent consistency at $z=3$ holds.

4. Self-regulating AGN feedback leads to better agreement between the two methods on the thermal state of the halo gas. Regardless of the numerical method, such an AGN will accrete matter until moving most of the gas to entropies of roughly $100 \mathrm{keV} \mathrm{cm}^{2}$, at which point subsequent accretion diminishes.

5. We see hints of AGN feedback impacting the termination point and orientation of filamentary cold flows in AMR, acting to push these streams beyond the impact radius of the AGN. In SPH, the filaments are more discrete, allowing the AGN-heated gas to escape around the flows, with little impact on their location or orientation. This may also explain the decrease in angular momentum of the central halo in AMR.

While the consistency between AMR and SPH seen here for AGN simulations is encouraging, we admit certain caveats. First, these results are for a single implementation of AGN feedback, but, as discussed in Section 1, there are a wide variety of numerical models being employed that include the impact of AGN feedback on galaxy evolution. Future work would benefit from a broader comparison study between AMR and SPH, as well as moving-mesh codes. Additionally, the agreement seen here appears to be due to the development of a self-regulation scenario. However, other feedback models that more accurately resolve the clumpy structure on small scales near the AGN could lead to less global heating of the halo environment due to shadowing, which could avert selfregulation. It is unclear how well the two simulation methods would agree when self-regulation does not occur.

Finally, the critical entropy value of $100 \mathrm{keV} \mathrm{cm}{ }^{2}$ is consistent with a non-cool core cluster. It is possible that our model omits the possibility of a cool-core cluster, with a central entropy value of roughly $30 \mathrm{keV} \mathrm{cm}{ }^{2}$ and a cooling time less than the Hubble time, where observations suggest AGN feedback via the radio mode, also known as the kinetic or maintenance mode, is able to maintain a heating rate comparable to the cooling rate (e.g., Burns 1990, and see Peterson \& Fabian 2006; McNamara \& Nulsen 2007; Cattaneo et al. 2009, and Fabian 2012 for reviews). In this work, at low accretion rates we continue to use an isotropic quasar-mode feedback model, which may prevent such a maintenance mode from occurring. Future work comparing a dual mode model that implements a radio-mode prescription at low accretion rates is needed. However, recent work by Hahn et al. (2015) suggests a cosmic origin for the non-cool core/cool core dichotomy, where low angular momentum major mergers disrupt the cool cores. This would suggest that a large sample of simulated galaxy clusters must be studied to determine how the two simulation methods compare for both cool-core and non-cool core clusters.

In summary, AGNs as modeled here can clearly play an important role in the evolution and regulation of cluster growth. Their possible observational impact is becoming clearer as surveys become larger and hydrodynamic simulations become more complex. Further work exploring the detailed physical implications of AGN feedback and its interaction with the 

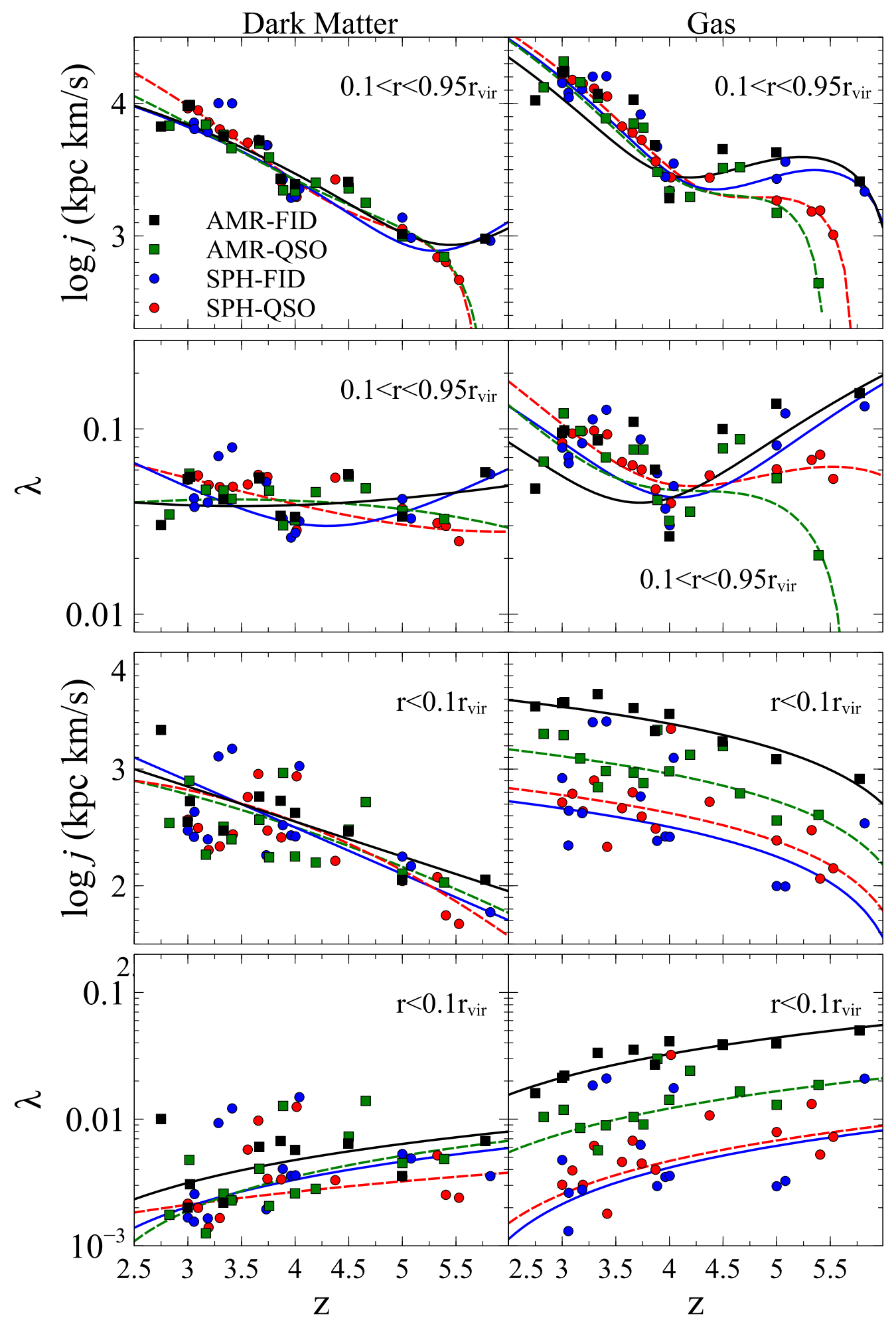

Figure 19. Top two rows: evolution of the specific angular momentum (top row) and spin parameter (second row) of the cluster dark matter (left) and gas (right) outside $0.1 r_{\text {vir }}$. Bottom two rows: evolution of the specific angular momentum (third row) and spin parameter (bottom row) of the cluster dark matter (left) and gas (right) within $0.1 r_{\mathrm{vir}}$. An approximate fit to the AMR-FID (SPH-FID) simulation is presented with solid black (blue) lines where we mark the measured values in an output by squares (circles). An approximate fit to the AMR-QSO (SPH-QSO) simulation is presented with dashed green (red) lines.

environments is essential for understanding the cosmic history of the universe.

This research has been supported by the Balzan Foundation via the University of Oxford. M.L.A.R. was supported by NSF grant AST11-03608 and the National Science and Engineering
Research Council of Canada. E.S. was also supported by the National Science Foundation under grant AST11-03608 and NASA theory grants NNX09AD106 and NNX15AK826. R.J. T. is supported by a Discovery Grant from NSERC, the Canada Foundation for Innovation, the Nova Scotia Research and 


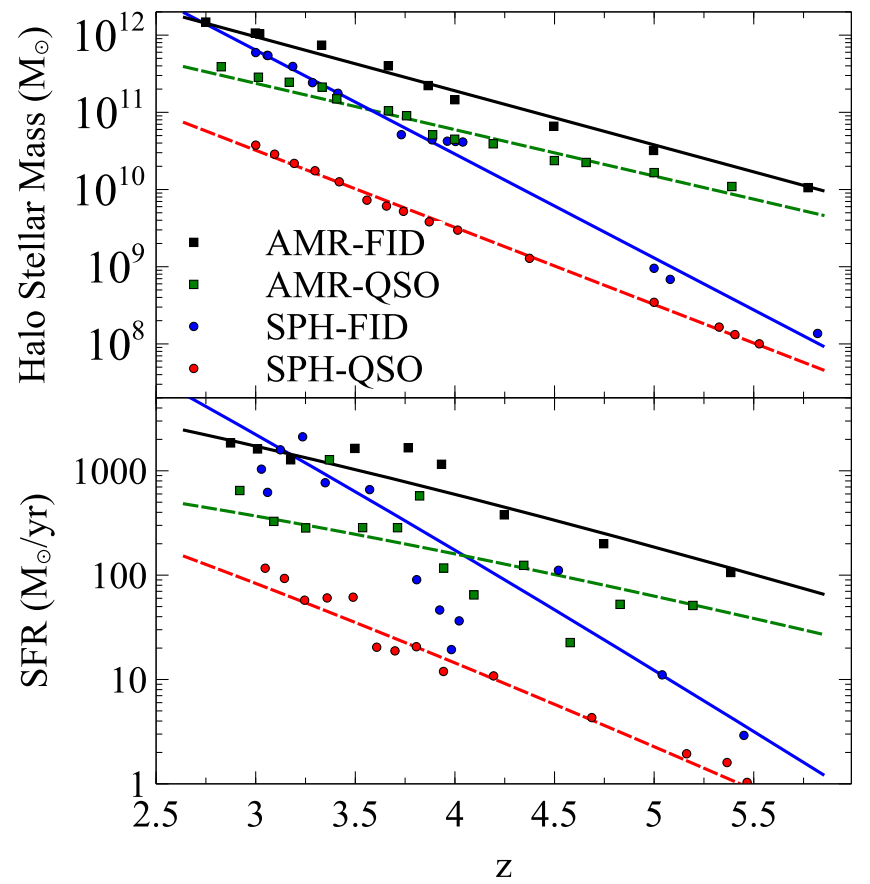

Figure 20. Top: evolution of the halo stellar mass of the cluster. The AMR-FID (SPH-FID) simulation is presented with a solid black (blue) line where we mark the measured values in an output by squares (circles). The AMR-QSO (SPH-QSO) simulation is presented with a dashed green (red) line. Lines are fitted exponential laws of the same form as Equation (4). Bottom: evolution of the halo star formation rate, taken as the change in halo stellar mass between adjacent outputs. Lines, given by the derivative of the exponential fits in the top plot, follow the same color scheme.

Table 3

Halo Stellar Mass Fitting Parameters

\begin{tabular}{lcc}
\hline \hline Run & $M_{3 *}$ & $\alpha_{*}$ \\
\hline AMR-FID & $9.9 \times 10^{11} M_{\odot}$ & 1.6 \\
AMR-QSO & $2.2 \times 10^{11} M_{\odot}$ & 1.4 \\
SPH-FID & $6.4 \times 10^{11} M_{\odot}$ & 3.1 \\
SPH-QSO & $3.2 \times 10^{10} M_{\odot}$ & 2.3 \\
\hline
\end{tabular}

Innovation Trust, and the Canada Research Chairs Program. Simulations were conducted with the CFI-NSRIT funded St. Mary's Computational Astrophysics Laboratory, and the High Performance Computing environment at the University of Leicester. M.L.A.R. would also like to thank Debora Sijacki for very insightful discussions, and the International Balzan Prize Foundation for contributing to the breadth of this work.

\section{REFERENCES}

Agertz, O., Moore, B., Stadel, J., et al. 2007, MNRAS, 380, 963

Arav, N., Borguet, B., Chamberlain, C., Edmonds, D., \& Danforth, C. 2013, MNRAS, 436, 3286

Barai, P., Viel, M., Murante, g., Gaspari, m., \& Borgani, S. 2014, MNRAS, 437, 1456

Bauer, A., \& Springel, V. 2012, MNRAS, 423, 2558

Bauer, F. E., Fabian, A. C., Sanders, J. S., Allen, S. W., \& Johnstone, R. M. 2005, MNRAS, 359, 1481

Benson, A. J., Bower, R. G., Frenk, C. S., et al. 2003, ApJ, 599, 38

Bett, P., Eke, V., Frenk, C. S., et al. 2007, MNRAS, 376, 215

Birdsall, C. K., \& Fuss, D. 1997, JCoPh, 135, 141

Birnboim, Y., \& Dekel, A. 2003, MNRAS, 345, 349

Blondin, J. M., Wright, E. B., Borkowski, K. J., \& Reynolds, S. P. 1998, ApJ, 500,342

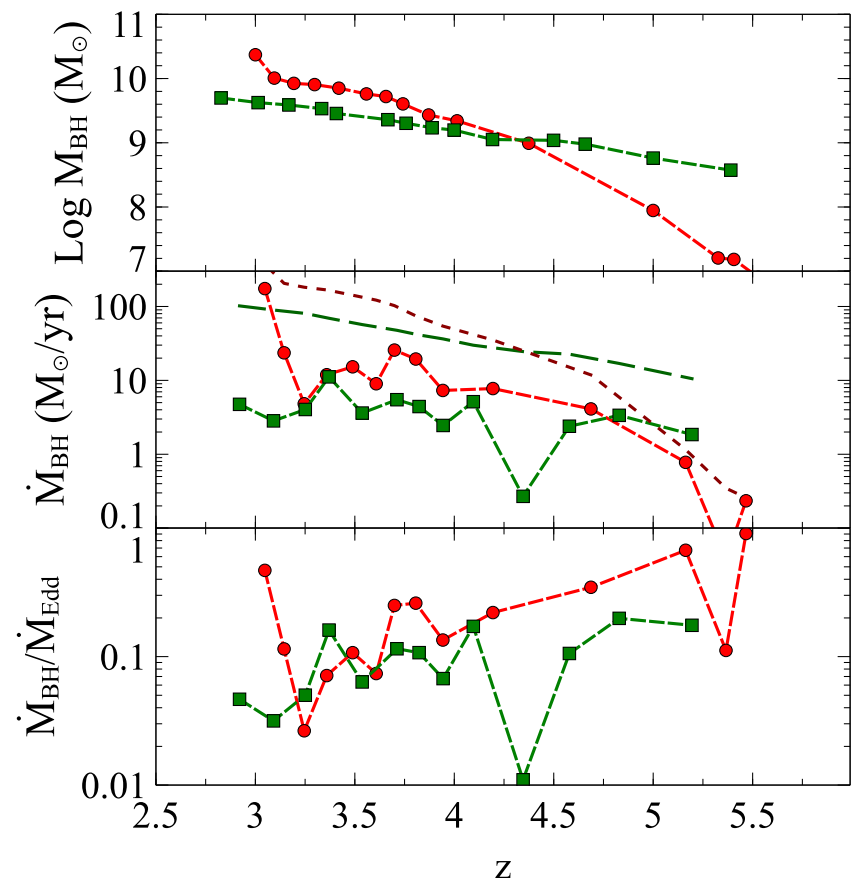

Figure 21. Top: evolution of the sum of the mass of the halo's three most massive black holes. The AMR-QSO (SPH-QSO) simulation is presented with a dashed green (red) line, where we mark the measured values in an output by squares (circles). Middle: evolution of the total growth rate of the three most massive halo black holes, taken as the change in mass between adjacent outputs, and thus includes mass growth due to merging. Lines follow the same color scheme as above. The dark green long-dashed (dark red dotted) line indicates the sum of the Eddington accretion rates for these three AMR-QSO (SPH-QSO) black holes. Bottom: same as middle, but normalized by the Eddington accretion rate sum.

Bondi, H. 1952, MNRAS, 112, 195

Booth, C. M., \& Schaye, J. 2009, MNRAS, 398, 53

Brinchmann, J, \& Ellis, R. 2000, ApJL, 536, L77

Bullock, J. S., Dekel, A., Kolatt, T. S., et al. 2001, ApJ, 555, 240

Burns, J. O. 1990, AJ, 99, 14

Cattaneo, A., Faber, S. M., Binney, J., Dekel, A., \& Kormendy, J. 2009, Natur, 460,213

Cavagnolo, K. W., Donahue, M., Voit, G. M., \& Sun, M. 2009, ApJS, 182, 12 Chapon, D., Mayer, L., \& Teyssier, R. 2013, MNRAS, 429, 3114

Couchman, H. M. P. 1991, ApJL, 368, L23

Couchman, H. M. P., Thomas, P. A., \& Pearce, F. R. 1995, ApJ, 452, 797

Cowie, L. L., Songaila, A., Hu, E. M., \& Cohen, J. G. 1996, ApJ, 112, 3

Croton, D. J., Volker, S., White., S. D., et al. 2006, MNRAS, 365, 11

Danovich, M, Dekel, A., Hahn, O., Ceverino, D., \& Primack, J. 2015, MNRAS, 449, 2087

Di Matteo, T., Khandai, N., DeGraf, C., et al. 2012, ApJ, 745, 29

Dubois, Y., Devriendt, J., Slyz, A., \& Teyssier, R. 2010, MNRAS, 409, 985

Dubois, Y., Devriendt, J., Slyz, A., \& Teyssier, R. 2012, MNRAS, 420, 2662

Dubois, Y., Pichon, C., Devriendt, J., et al. 2013, MNRAS, 428, 2885

Dubois, Y., \& Teyssier, R. 2008, A\&A, 477, 79

Dunn, R. J. H., \& Fabian, A. C. 2006, MNRAS, 373, 959

Dunn, R. J. H., Fabian, A. C., \& Celotti, A. 2006, MNRAS, 372, 1741

Einfeldt, B. 1988, SJNA, 25, 294

Fabian, A. C. 1994, ARA\&A, 32, 277

Fabian, A. C. 2012, ARA\&A, 50, 455

Fabian, A. C., \& Nulsen, P. E. J. 1977, MNRAS, 180, 479

Falcke, H, \& Biermann, P. L. 1999, A\&A, 342, 49

Ferrarese, L., \& Merritt, D. 2000, ApJ, 539, 9

Frenk, C. S., White, S. D. M., Bode, P., et al. 1999, ApJ, 525, 554

Gebhardt, K., Bender, R., Bower, G., et al. 2000, ApJ, 539, 13

Genel, S., Fall, S. M., Hernquist, L., et al. 2015, ApJL, 804, L40

Gerritsen, J. P. E., \& Icke, V. 1997, A\&A, 325, 972

Gingold, R. A., \& Monaghan, J. J. 1977, MNRAS, 181, 375

Guzman, R., Gallego, J., Koo, D. C., et al. 1997, ApJ, 489, 559

Haardt, F., \& Madau, P. 1996, ApJ, 461, 20

Hahn, O., Martizzi, D., Hao-Yi, W., et al. 2015, MNRAS, arXiv:1509.04289 
Hayward, C. C., Torrey, P., Springel, V., Hernquist, L., \& Vogelsberger, M. 2014, MNRAS, 442, 1992

Heavens, A., Panter, B., Jimenez, R., \& Dunlop, J. 2004, Natur, 428, 625

Hockney, R. W., \& Eastwood, J. W. 1981, Computer Simulation Using Particles (New York, NY: McGraw-Hill)

Hopkins, A. M., \& Beacom, J. F. 2006, ApJ, 651, 142

Hopkins, P. F. 2013, MNRAS, 428, 2840

Hubber, D. A., Falle, S. A. E. G., \& Goodwin, S. P. 2013, MNRAS, 432, 711

Karim, A., Schinnerer, E., Martínez-Sansigré, A., et al. 2011, ApJ, 730, 61

Kaufman, T., Mayer, L., Wadsley, J., Stadel, J., \& Moore, B. 2006, MNRAS, 370,1612

Kaufman, T., Mayer, L., Wadsley, J., Stadel, J., \& Moore, B. 2007, MNRAS, 375, 53

Kennicut, R. C. 1998, ApJ, 498, 541

Kereš, D., Vogelsberger, M., Sijacki, D., Springel, V., \& Hernquist, L. 2012, MNRAS, 425, 2027

Kim, J., Abel, T., Agertz, O., et al. 2014, ApJS, 210, 14

Komatsu, E., Smith, K. M., Dunkley, J., et al. 2011, ApJS, 192, 18

Krumholz, M. R., \& Tan, J. C. 2007, ApJ, 654, 304

Larson, D., Dunkley, J., Hinshaw, G., et al. 2011, ApJS, 192, 16

Le Brun, A. M. C., McCarthy, I. G., Schaye, J., \& Trevor, P. J. 2014, MNRAS, 441,1270

Loewenstein, M. 2004, in Origin and Evolution of the Elements, ed. A. McWilliam, \& M. Rauch (Cambridge: Cambridge Univ. Press), 422

Lucy, L. B. 1977, AJ, 82, 1013

Maiolino, R., Gallerani, S., Neri, R., et al. 2012, MNRAS, 425, L66

Marri, S., \& White, S. D. M. 2003, MNRAS, 345, 561

Martizzi, D., Teyssier, R., \& Moore, B. 2013, MNRAS, 432, 1947

McCarthy, I. G., Schaye, J., Ponman, T. J., et al. 2010, MNRAS, 406, 822

McNamara, B, \& Nulsen, P. E. J. 2007, ARA\&A, 45, 117

Merloni, A., \& Heinz, S. 2007, MNRAS, 381, 589

Mitchell, N. L., McCarthy, I. G., Bower, R. G., Theuns, T., \& Crain, R. A. 2009, MNRAS, 395, 180

Moe, M., Arav, N., Bautista, M. A., \& Korista, K. T. 2009, ApJ, 706, 525

Morris, J. P. 1996, PASA, 13, 97

Nelson, D., Genel, S., Vogelsberger, M., et al. 2015, MNRAS, 448, 59

Nelson, D., Vogelsberger, M., Genel, S., et al. 2013, MNRAS, 429, 3353

Nulsen, P. E. J., Stewart, G. C., Fabian, A. C., et al. 1982, MNRAS, 1999, 1089

Oh, S. P., \& Benson, A. 2003, MNRAS, 342, 664

Okamoto, T., Jenkins, A., Eke, V. R., Quilis, V., \& Frenk, C. S. 2003 , MNRAS, 345, 429

O’Shea, B. W., Nagamine, K., Springel, V., Henquist, L., \& Norman, M. L. 2005, ApJS, 160, 1

Ostriker, E. C. 1999, ApJ, 513, 252

Panter, B., Jimenez, R., Heavens, A. F., \& Charlot, S. 2007, MNRAS, 378, 1550

Peterson, J. R., \& Fabian, A. C. 2006, PhR, 427, 1

Peterson, J. R., Paerels, F. B. S., Kaastra, J. S., et al. 2001, A\&A, 365, L104

Pike, S. R., Kay, S. T., Newton, R. D. A., Thomas, P. A., \& Jenkins, J. 2014, MNRAS, 445, 1774

Planelles, S., Borgani, S., Fabjan, D., et al. 2014, MNRAS, 438, 195

Power, C., Read, J. I., \& Hobbs, A. 2014, MNRAS, 440, 3243
Price, D. J. 2012, JCoPh, 231, 759

Prunet, S., Pichon, C., Aubert, D., et al. 2008, ApJS, 178, 179

Rafferty, D. A., McNamara, B. R., Nulsen, P. E. J., \& Wise, M. W. 2006, ApJ, 652,216

Rasera, Y., \& Teyssier, R. 2006, A\&A, 445, 1

Rees, M. J. 1984, ARA\&A, 22, 471

Richardson, M. L. A., Scannapieco, E., \& Thacker, R. J. 2013, ApJ, 771, 81

Rosen, A., \& Bregman, J. N. 1995, ApJ, 440, 634

Sambruna, R. M., Aharonian, F. A., Krawczynski, H., et al. 2000, ApJ, 538,127

Scannapieco, C., Wadepuhl, M., Parry, O. H., et al. 2012, MNRAS, 423, 1726

Scannapieco, E., \& Oh, S. P. 2004, ApJ, 608, 62

Scannapieco, E., Silk, J., \& Bouwens, R. 2005, ApJ, 635, 13

Schmidt, M. 1959, ApJ, 129, 243

Sembolini, F., Elahi, P. J., Pearce, F. R., et al. 2016b, MNRAS, 459, 2973

Sembolini, F., Yepes, G., Pearce, F. R., et al. 2016a, MNRAS, 457, 4063

Shakura, N. I., \& Sunyaev, R. A. 1973, A\&A, 24, 337

Sijacki, D., Springel, V., Di Matteo, T., \& Hernquist, L. 2007, MNRAS, 380,877

Sijacki, D., Vogelsberger, M., Kereš, D., Springel, V., \& Hernquist, L. 2012, MNRAS, 424, 2999

Silk, J, \& Rees, M. J. 1998, A\&A, 331, L1

Sommer-Larsen, J., Gelato, S., \& Vedel, H. 1999, ApJ, 519, 501

Spacek, A., Scannapieco, E., Cohen, S., Joshi, B., \& Mauskopf, P. 2016, ApJ, 819,128

Spergel, D. N., Bean, R., Doré, O., et al. 2007, ApJS, 107, 377

Springel, V. 2005, MNRAS, 364, 1105

Springel, V. 2010, MNRAS, 401, 791

Springel, V., Di Matteo, T., \& Hernquist, L. 2005, MNRAS, 361, 776

Stewart, G. C., Fabian, A. C., Jones, C., \& Forman, W. 1984, ApJ, 285, 1

Sutherland, R. S., \& Dopita, M. A. 1993, ApJS, 88, 253

Tamura, T., Kaastra, J. S., Peterson, J. R., et al. 2001, A\&A, 365, L87

Teyssier, R. 2002, A\&A, 385, 337

Thacker, R. J., \& Couchman, H. M. P. 2000, ApJ, 545, 728

Thacker, R. J., \& Couchman, H. M. P. 2006, CoPhC, 174, 540

Thacker, R. J., Scannapieco, E., \& Couchman, H. M. P. 2006, ApJ, 653, 86

Thacker, R. J., Scannapieco, E., Couchman, H. M. P., \& Richardson, M. L. A. 2009, ApJ, 693, 552

Thomas, P. A., \& Couchman, H. M. P. 1992, MNRAS, 257, 11

Torrey, P., Vogelsberger, M., Sijacki, D., Springel, V., \& Hernquist, L. 2012, MNRAS, 427, 2224

Tremaine, S., Gebhardt, K., Bender, R., et al. 2002, ApJ, 574, 740

Turk, M. J., Smith, B. D., Oishi, J. S., et al. 2011, ApJS, 192, 9

van Leer, B. 1979, JCoPh, 32, 101

Vogelsberger, M., Sijacki, D., Kereš, D., Springel, V., \& Hernquist, L. 2012, MNRAS, 425, 3024

Voit, G. M., Kay, S. T., \& Bryan, G. L. 2005, MNRAS, 364, 909

Wechsler, R. H., Bullock, J. S., Primack, J. R., Kravtsov, A. V., \& Dekel, A. 2002, ApJ, 568, 52

White, S. D. M. 1984, ApJ, 286, 38

White, S. D. M., \& Rees, M. J. 1978, MNRAS, 183, 341

Wurster, J., \& Thacker, R. J. 2013, MNRAS, 431, 2513 